\title{
Artefact
}

Techniques, histoire et sciences humaines

\section{7 | 2017}

Os, bois, ivoire et corne : l'exploitation des matières dures d'origine animale

\section{De l'Atlantique à l'Oural : l'exploitation des matières osseuses au Paléolithique}

From the Atlantic to the Urals: Osseous Materials Exploitation during the Paleolithic

\section{Nejma Goutas, Marianne Christensen et Aline Averbouh}

\section{(2penEdition}

\section{Journals}

Édition électronique

URL : http://journals.openedition.org/artefact/989

DOI : $10.4000 /$ artefact. 989

ISSN : 2606-9245

\section{Éditeur :}

Association Artefact. Techniques histoire et sciences humaines, Presses universitaires du Midi

\section{Édition imprimée}

Date de publication : 30 mai 2018

Pagination : 9-38

ISBN : 978-2-7535-7494-6

ISSN : 2273-0753

Référence électronique

Nejma Goutas, Marianne Christensen et Aline Averbouh, « De l'Atlantique à l'Oural : I'exploitation des matières osseuses au Paléolithique », Artefact [En ligne], 7 | 2017, mis en ligne le 11 décembre 2018, consulté le 10 décembre 2020. URL : http://journals.openedition.org/artefact/989; DOI : https:// doi.org/10.4000/artefact.989

\section{(c) (i) $\ominus$}

Artefact, Techniques, histoire et sciences humaines est mise à disposition selon les termes de la Licence Creative Commons Attribution - Pas d'Utilisation Commerciale - Pas de Modification 4.0 International. 


\title{
De l'Atlantique à l'Oural : I'exploitation des matières osseuses au Paléolithique
}

\author{
Nejma GOUTAS* \\ Marianne CHRISTENSEN** \\ Aline AVERBOUH ${ }^{* * *}$
}

\section{Résumé}

Le travail des matières osseuses est connu ponctuellement dès le Pléistocène moyen à travers de simples éclats en os à peine modifiés notamment en Europe (Espagne, Italie et Allemagne), mais aussi au Proche-Orient (Israël). C'est seulement à la fin du Pléistocène récent qu'émerge une véritable économie des matières dures animales,

*. Nejma Goutas est chargée de recherche au CNRS, équipe Ethnologie Préhistorique (UMR 7041). Préhistorienne, spécialiste du Paléolithique supérieur ancien et des industries en matières dures d'origine animale, ses recherches se concentrent principalement sur le Gravettien (30-20 Ka non cal BP), en Europe (France, Belgique, Moravie, Russie, Roumanie). Elle a publié : « De Brassempouy à Kostienki : l'exploitation technique des ressources animales dans l'Europe gravettienne », in Marcel OtтE (dir.), Les Gravettiens, Paris, éd. Errance, 2013, p. 105-160; « From stone flaking to grinding: Three original Pavlovian antler tools from Moravia (Pavlov I, Czech Republic) », Quaternary International, vol. 359-360, 2015, p. $240-260$. Contact : [nejma.goutas@cnrs.fr].

**. Marianne Christensen est maître de conférences, université de Paris 1 Panthéon-Sorbonne, équipe Ethnologie Préhistorique (UMR 7041). Spécialiste de l'équipement en matières dures d'origine animale des sociétés européennes de chasseurs-cueilleurs et de l'extrême sud du continent américain (Patagonie), elle a publié : L'industrie osseuse des chasseurs-cueilleurs : le cas des nomades marins de Patagonie et Terre de Feu, Punta Arenas, ediciones Universidad de Magallanes, colección Poblamiento Humano de FuegoPatagonia, 2016 et, avec José-Miguel TEJERo, « La fabrication d'objets en matières dures animales », in Marie Balasse, Jean-Philippe Brugal, Yannick Dauphin, Eva Maria Geigl, Christine Oberlin et Ina Reiche (dir.), Messages d'os. Archéométrie du squelette animal et humain, Paris, Éditions des archives contemporaines, coll. Sciences Archéologiques, 2015, p. 73-90. Contact : [marianne.christensen@univ-paris1.fr].

***. Chargée de recherche au CNRS, Unité Mixte de Recherche «Archéozoologie, archéobotanique : sociétés, pratiques et environnement », Muséum National d'Histoire Naturelle de Paris, Aline Averbouh est préhistorienne, spécialiste du Paléolithique supérieur et de la transition avec le Mésolithique, et de l'étude de la fabrication et de la constitution des équipements en matières dures d'origine animale, particulièrement pour les cultures magdaléniennes (ancienne à finale), aziliennes et laboriennes d'Europe occidentale. Elle a publié : « Le travail des matières osseuses et les productions associées », in Michèle Julien et Claudine Karlin (dir.), Un automne à Pincevent. Le campement magdalénien du niveau IV20, Paris, Société préhistorique française, mémoire 57, 2014, p. 151-186; et, avec Peggy JAcQuEMENT et Jean-Jacques Cleyet-Merle (dir.), L'A quitaine à la fin des temps glaciaires. Les sociétés de la transition du Paléolithique final au début du Mésolithique dans l'espace nord aquitain. Hommage à Guy Célérier, musée national de Préhistoire, Les Eyzies de Tayac, juin 2015, hors série Paleo, sous presse. Contact : [aline.averbouh@mnhn.fr]. 
avec son lot d'innovations techniques et conceptuelles aboutissant à une étonnante diversité des produits. Ce changement est enregistré à différents moments : il apparaît sur le continent européen à partir du Paléolithique supérieur, en même temps que l'arrivée de l'homme anatomiquement moderne, il y a près de 40000 ans, alors que sur le continent africain de véritables industries osseuses, fabriquées aussi par des hommes anatomiquement modernes, sont connues 35000 ans plus tôt, dès le Middle Stone Age. Ce sont les grandes étapes de cette longue et complexe histoire de l'exploitation technique des ressources animales sur le continent européen (principalement en Europe occidentale) que nous retracerons ici, en nous arrêtant, au préalable, sur celles qui ont structuré le cadre méthodologique des études sur les industries osseuses paléolithiques.

Mots-clefs : Europe, industries osseuses, innovations techniques et symboliques, Paléolithique.

\section{Abstract. From the Atlantic to the Urals: Osseous Mate- rials Exploitation during the Paleolithic}

Barely modified bone flakes reflect occasional work of osseous materials since the Middle Pleistocene in Europe (Spain, Italy, Germany), but also in the Near East (Israel). Only at the end of Early Pleistocene a clear economy of hard animal materiel appears with both technical and conceptual innovation leading to a rich variety of osseous products. This change occurred in various places at different time: in Europe it goes together with the arrival of anatomical modern humans some 40000 years ago; whereas in Africa true osseous industries are known 35000 years earlier dated to the Middle Stone Age. The paper will highlight this long and complicates history of technical processing of animal resources in Europe (mainly Western Europe), starting with the major steps that have structured the methodological framework for the study of osseous industries.

Key words: Europe, Paleolithic, osseous industry, technical and symbolic innovations.

À la fin du xIx siècle, l'un des principaux objectifs de l'étude de l'industrie osseuse était d'appréhender la fonction des instruments et des armes, et donc indirectement correspondait à un intérêt orienté vers les activités de ces hommes préhistoriques tout juste reconnus en tant qu'êtres humains. Le comparatisme ethnographique, avec les instruments « d'autres êtres sauvages », a donné lieu aux premières nomenclatures fonctionnelles dont beaucoup de termes sont encore en usage. Le début $\mathrm{du} \mathrm{xx}^{\mathrm{e}}$ siècle voit les premiers classements d'objets fondés sur la morphologie de la partie supposée active, proposant des classes 
d'outils servant à couper, à percer ${ }^{1} \ldots$ mais aussi des études ponctuelles, dont certaines font déjà appel à l'approche expérimentale (en France ${ }^{2}$, dès le début du $x^{\mathrm{e}}$ siècle ou en Union soviétique ${ }^{3}$, à partir des années 1930-1940).

Les années 1970 furent particulièrement fécondes pour les études sur les industries en matières dures d'origine animale (MDA). Ce sont notamment les deux premiers colloques sur l'industrie osseuse préhistorique, organisés en 1974 et 1976 par Henriette CampsFabrer, qui permettent d'instaurer une véritable approche disciplinaire. Force est de constater que le développement tardif de ces études a nécessairement généré un retard de connaissances dans ce domaine. Quelques décennies d'existence et de mise à l'épreuve ne peuvent suffire à compenser le nombre d'études et de synthèses encore nécessaires pour atteindre la résolution désormais offerte par l'étude des industries lithiques. Pour autant, cet écueil est surtout contextuel et non (plus) structurel, puisque depuis les années 1970-1980, l'étude des industries en matières dures d'origine animale s'est progressivement dotée d'outils analytiques des plus pertinents, sur le plan typologique (une nomenclature neutre) et technologique (avec la constitution de référentiels de stigmates techniques, de traces taphonomiques et d'usures fonctionnelles, de remontages mentaux ${ }^{4}$ et physiques). Pour beaucoup, ces outils méthodologiques se sont inspirés de la technologie lithique, tout en s'adaptant aux particularités des matières dures d'origine animale. La première de ses particularités réside dans la fraction organique de ces ressources, fraction vouée à se transformer et à disparaître.
Cela induit nécessairement des biais dans l'étude du mobilier osseux, allant de légères altérations de surface et de volume jusqu'à la destruction totale de l'artefact. Penser la culture matérielle en raisonnant aussi sur l'absence n'a rien de très nouveau, mais en technologie osseuse, cette absence n'est jamais univoque; elle peut tout autant refléter des choix humains (désintérêt pour ces matières, segmentation de la chaîne opératoire dans le temps et l'espace...), que des interventions post-dépositionnelles (effacement partiel ou total des stigmates techniques ou fonctionnels sous l'action de processus taphonomiques variés).

Et c'est donc avec cette difficulté supplémentaire qu'il nous faut composer surtout lorsque l'on travaille sur les productions osseuses du Paléolithique, nécessairement davantage soumises à ces biais de conservation, compte tenu de leur ancienneté. 


\section{La typologie comme outil de classification des formes...}

Les équipements en matières dures animales ont très tôt, dès la fin du XIX $^{\mathrm{e}}$ siècle (travaux de Gabriel et Adrien de Mortillet, par exemple) bénéficié d'une approche strictement classificatoire des formes. Cette approche, en son temps, avait permis de construire un premier cadre d'étude essentiel pour la compréhension de ces industries. L'intérêt pour la forme des objets archéologiques et leurs décors prime alors sur la connaissance des savoir-faire en jeu dans leur acquisition et leur transformation et devient le principal outil pour l'élaboration des sériations chrono-culturelles (Fig. I, cahier couleur). Cette conception de la typologie marquera longtemps la recherche en archéologie préhistorique, en France, comme en Europe ${ }^{5}$. En classant les objets archéologiques selon des attributs de matière, de formes et de dimensions, la typologie aura été, et continue d'être, un outil très utile pour ordonner des faits, afin d'appréhender de manière commune les assemblages (comparaison inter ou intra-site) et être ainsi en mesure d'identifier des différences ou des parentés dans les activités de fabrication et de consommation. Cette utilisation de la typologie, dans une dimension somme toute assez restrictive (cf. infra) et éloignée de sa définition première, n'en fut pas moins un jalon nécessaire de la construction du discours scientifique, et en ce sens, il serait anachronique $\mathrm{d}^{\prime} \mathrm{y}$ porter une critique dépréciative.

\section{La technologie au service de la typologie - un autre regard sur les industries osseuses}

Avec la prise en compte des déchets et des ratés de fabrication, essentiellement à partir des années 1970, un basculement profond s'est opéré dans les années 1990-2000, donnant lieu à l'élaboration de " typologies à ambition interprétative » recherchant des «normes de comportements » et in fine des "valeurs", en intégrant pleinement les données technologiques (depuis l'acquisition de la matière jusqu'à son utilisation ${ }^{6}$ ). Ces nouvelles recherches fondées sur une approche technologique des industries osseuses ont permis de mettre en lumière de nouveaux critères et marqueurs techniques, parfois invisibles à l'œil nu, et qui sont autant de signatures culturelles discrètes de manières de concevoir une forme, une matière, une fonction ou une action. Dès lors, le regard du Préhistorien se modifie, puisque la forme d'un objet archéologique n'est plus pensée pour elle-même, mais à la fois comme la conséquence d'un « [...] besoin (objectif fonctionnel), d'un cycle de vie (utilisation, affûtage, réfection, recyclage), d'une action sur la matière (choix et ordonnancement des 
techniques opérés), des propriétés intrinsèques des matériaux exploités autant que des modalités de leur approvisionnement ${ }^{7}$ ». L'approche typologique a ainsi renoué avec sa définition première, celle d'une science à part entière de l'analyse et de la description des formes de quelque nature qu'elle soit ${ }^{8}$. Elle peut (et devrait) tout autant être appliquée aux choix d'approvisionnement, aux méthodes de taille, qu'aux modes de fabrication et d'utilisation des instruments. ${ }^{9}$. Les travaux d'Aline Averbouh sur l'exploitation du bois de renne au Magdalénien sont ainsi les premiers à proposer, pour le Paléolithique, une classification des déchets de fabrication (matrices, chutes) fondée sur la reconnaissance de savoir-faire particuliers, guidés par des normes culturelles très fortes, au point que les déchets, euxmêmes, répondent à une forte standardisation. Ces travaux démontrent que les déchets de fabrication peuvent offrir des éléments de diagnoses chrono-culturelles aussi pertinents que les attributs de forme des objets finis ${ }^{10}$.

La pertinence de ce changement de paradigme s'illustre aussi dans la distinction que l'approche technologique aura permis de faire entre outil et déchet de fabrication. C'est le cas de pointes en bois de cervidé dites "à base raccourcie ${ }^{11}$ " ou à " base mâchonnée ${ }^{12}$ ", longtemps considérées comme des pointes de projectiles, et dont l'étude recontextualisée au sein d'un processus d'exploitation général a permis de démontrer qu'elles étaient des déchets en lien avec la fabrication d'objets appointés ${ }^{13}$. Cet exemple illustre bien les limites de la typologie descriptive, dès lors qu'elle occulte l'objectif techno-fonctionnel qui sous-tend la forme d'un artefact. Cette distinction parfois subtile, entre outil et déchet en contexte préhistorique, tient aussi au fait qu'un même objet peut intégrer ces deux statuts. C'est le cas des «matricesoutils » en bois de cervidé, identifiées en contexte gravettien ${ }^{14}$. Dans certains cas, il s'agit de la récupération d'un déchet (les matrices) en vue de sa transformation en outil biseauté. Dans d'autres cas, ces matrices-outils reflètent une gestion plus complexe de la matière première puisqu'en une seule opération (l'extraction d'une baguette), le fabricant crée à la fois la préforme très aboutie d'un outil biseauté et extrait un support à la forme et aux dimensions désirées.

L'approche technologique couplée à l'approche fonctionnelle a en outre permis de repenser la typologie des outils de transformation en mettant en lumière des outils jusqu'alors minorés, comme les outils intermédiaires bruts sur éclats diaphysaires ${ }^{15}$. Cet outillage dit " non façonné $e^{16}$ », «brut ${ }^{17}$ » ou «peu élaboré ${ }^{18}$ » (sans aucune connotation péjorative) est présent dans l'équipement tout au long du Paléolithique supérieur. Les supports osseux sont judicieusement sélectionnés parmi les restes alimentaires ou intentionnellement débités pour offrir des formes immédiatement fonctionnelles ou façonnées a minima (cf. infra). 


\section{La technologie, une approche consubstantielle de la palethnologie... une palethnologie au service d'une ambition paléohistorique}

La technologie osseuse, pas plus que celle d'autres matériaux (lithique, céramique, métal, bois végétal, etc.) n'est une finalité en soi. Son intérêt réside dans le fait que cette approche offre des moyens objectifs et objectivables de replacer un objet archéologique dans une dimension anthropologique... Cette dernière est toutefois toujours incomplète, car si nous sommes en mesure de dire comment un objet est fabriqué et utilisé, les valeurs, les croyances idéologiques, symboliques, qui sous-tendent sa place dans le système qui lui a donné vie ${ }^{19}$, n'en demeurent pas moins inaccessibles en archéologie préhistorique. Quoiqu'il en soit, la technologie permet de dépasser les attributs de forme d'un objet pour s'intéresser à ce qui n'est souvent pas directement visible à l'œil nu. Le choix des matières, des techniques et leur articulation au sein d'un processus de fabrication et d'utilisation nous informent sur les représentations collectives en œuvre au sein d'une société. Dès lors, le partage entre deux groupes humains d'un même savoir-fair ${ }^{20}$ suppose l'existence d'interactions sociales, car son apprentissage et sa reproduction n'est possible que s'il est porté par un réseau social assurant la transmission des compétences ${ }^{21}$. L'approche technologique, couplée à l'approche typologique, constitue donc un moyen privilégié pour rendre compte de dynamiques sociétales et historiques sur le temps long, en un mot pour écrire une paléohistoire (sensu Valentin). La technologie est ainsi une approche des plus essentielles pour identifier des permanences, des changements ou des ruptures dans le temps et l'espace entre différents groupes humains.

Évaluer la pertinence et la signification des permanences, des changements et des ruptures que nous observons au sein de la culture matérielle est indissociable de la résolution d'analyse que nous utilisons. Plus la focale sera large, plus les variabilités intersites, inter-régions seront lissées. À l'inverse, plus la focale sera réduite, plus les variabilités seront visibles avec le risque de s'y perdre ou de les surinterpréter en termes de variations culturelles. De fait, le croisement des données de l'industrie osseuse avec celles de l'ensemble de la culture matérielle et idéelle (industries lithiques, arts mobilier et pariétal, pratiques funéraires, etc.) constitue assurément une voie $\mathrm{d}^{\prime}$ investigation émergente ${ }^{22}$ qu'il faut continuer de développer dans le futur. Il serait illusoire d'attendre que toutes ces données coïncident systématiquement, mais ce n'est que par ce type d'approche systémique que l'arythmie des phénomènes culturels peut être perçue, et par la suite questionnée ${ }^{23}$. 


\section{Les premières utilisations des matières dures d'origine animale en Europe}

\section{Au plus proche de la réalité grâce à la taphonomie}

Jusqu'à une époque assez récente, faute d'analyses techniques et taphonomiques précises, certaines pièces osseuses particulières obtenaient $\mathrm{d}^{\prime} \mathrm{em}$ blée le statut d'industrie. Ainsi l'abbé Breuil interpréta, dès les années 1930, des fragments d'os comme des restes d'activité technique sur le site de Choukou-tien ${ }^{24}$ en Chine, et vingt ans plus tard, Raymond Dart évoquait l'existence d'un stade " prélithique " chez les Australopithèques, fondé sur une utilisation technique d'ossements d'animaux $^{25}$. D'autres préhistoriens mènent des reconstitutions expérimentales pour démontrer le caractère anthropique des objets (par exemple Louis Leguay ${ }^{26}$, pour la gravure; Léon Henri-Martin ${ }^{27}$, pour la fracturation; ou Luis Siret ${ }^{28}$, pour les retouchoirs). La combinaison d'une lecture à la fois taphonomique et technique permettra de faire un "vrai nettoyage " dans ces « industries » osseuses anciennes, fabriquées parfois par l'homme, parfois par la nature. Ainsi, les recherches de Francesco $\mathrm{d}^{\prime}$ Errico et Paola Villa ${ }^{29}$ sur des os gravés du Paléolithique moyen conduiront à les écarter du domaine de l'art mobilier; des tracés géométriques initialement pris pour un travail artistique de gravure se révéleront être des empreintes naturelles de canaux vasculaires (Pech de l'Aze, Cueva Morin...). Ces chercheurs ont de la même façon ôté leur statut d'outils aux poinçons moustériens provenant de certains sites comme Vaufrey, Camiac ou encore Pech de l'Aze, car leur morphologie appointée résulterait de phénomènes sédimentaires et chimiques naturels ${ }^{30}$. Les pièces interprétées comme des projectiles sur extrémité distale de défense de proboscidiens de Torralba et d'Ambrona en Espagne résulteraient de fractures occasionnées lors de combats entre animaux ${ }^{31}$. Quant aux perforations de certains éclats d'os considérés comme des pendeloques moustériennes, elles découleraient d'une action chimique naturelle ${ }^{32}$. L'industrie osseuse de ces périodes anciennes s'est ainsi appauvrie, et se limite désormais à quelques objets peu transformés.

\section{De l'os à la coquille}

En Europe, l'utilisation de matières dures animales à des fins autres qu'alimentaires est documentée dès le Paléolithique inférieur ${ }^{33}$ (Fig. II, cahier couleur, $n^{\text {os }} \mathbf{1}$ et 2 ). Ces témoins constituent, en l'état actuel des recherches, des découvertes certes exceptionnelles mais isolées et très disparates dans le temps et l'espace. La majeure partie des pièces sont en os et peuvent le plus souvent être décrites avec une terminologie empruntée à l'industrie lithique, avec laquelle la parenté est évidente ( $c f$. infra). En effet, à cette époque, ainsi qu'au Paléolithique moyen, l'os est transformé en outil principalement par percussion et l'expression clairvoyante de "pierre animale » utilisée par l'abbé Breuil prend ici tout son sens $s^{34}$. 
Cette intégration précoce de l'os à la sphère technique, à la différence des bois de cervidés ou de l'ivoire, tient probablement à ses potentialités alimentaires. Ces deux objectifs - alimentaire et techniquesont souvent étroitement imbriqués et il est difficile de clairement les distinguer. Quoiqu'il en soit, l'exploitation de carcasses animales, et la récupération de la moelle en particulier, auraient permis aux premiers homininés d'éprouver les aptitudes et les contraintes de ce matériau à la fois solide et cassant, mais dont la fracturation, voire la «taille», peuvent être contrôlées pour offrir des tranchants aussi efficaces que ceux des outils en silex. Une utilisation directe de certains os (pour leurs volumes, leurs formes et leurs inerties) comme outils bruts n'est pas non plus à exclure (pour frapper, concasser ou broyer par exemple), même si elle demande à être clairement démontrée. De telles hypothèses ont d'ailleurs existé de longue date, en premier lieu sous la plume de l'abbé Breuil ${ }^{35}$ et de Pierre Teilhard de Chardin ${ }^{36}$, mais c'est sans nul doute la célèbre " industrie ostéondokératique » de Raymond Dart qui aura le plus marqué les esprits ( $c f$. supra).

$\mathrm{Au}$ Paléolithique inférieur, les outils les plus connus sont les bifaces façonnés notamment sur os d'éléphants antiques. Ils ont été découverts notamment sur trois sites italiens, en nombre très inégal, à Fontana Ranuccio (daté de 450000 uncal. BP), Castel di Guido et Malagrotta (datés $\mathrm{du}$ OIS 9$)^{37}$. Ces pièces ont en commun de présenter des stigmates caractéristiques de la fracture conchoïdale (négatifs d'enlèvement). Si l'on réfère aux rares exemplaires publiés, leurs formes sont loin d'être aléatoires et elles pré- sentent les mêmes recherches de symétrie que leurs homologues en pierre ${ }^{38}$, quoique à l'aide d'un nombre plus réduit $\mathrm{d}^{\prime}$ enlèvements ${ }^{39}$. Ces bifaces témoignent $\mathrm{d}^{\prime}$ une connaissance approfondie des propriétés structurelles de l'os. La densité des os choisis, autant que les propriétés " clastiques » de l'os auront permis aux homininés de transférer sur un nouveau matériau, un savoir-faire « inventé » sur matière minérale. Avec cette difficulté supplémentaire, sans nul doute, que l'os a une mémoire moins permissive aux erreurs que la pierre... un coup mal porté y imprime des fissures, des accidents, qui peuvent compromettre - de manière plus radicale - l'opération de taille. Si le façonnage de bifaces en os pourrait relever de l'anecdote préhistorique à l'échelle de la longue histoire du travail des matières dures animales, cette application au matériau osseux est, en réalité, un saut conceptuel majeur. En premier lieu car ce transfert n'est pas toujours une réponse à une absence de matière première lithique disponible localement (un choix par défaut en quelque sorte), mais traduit sans nul doute aussi une volonté de tester un concept volumétrique, une mise en forme complexe, une fonction, voire une esthétique ${ }^{40}$ sur une nouvelle matière. Quelles que furent ces motivations, elles auront conduit des groupes, peut-être différents mais appartenant à une même unité de temps (l'Acheuléen), à reproduire ce type d'outil en os sur différents gisements. D'autres objets en os retouchés, moins élaborés, ont aussi été retrouvés à La Polledraran Ceprano en Italie $^{41}$, à Bilzingsleben ${ }^{42}$ et à Schöningen ${ }^{43}$ en Allemagne, à Atapuerca ${ }^{44}$ en Espagne ou à Verteszöllös ${ }^{45}$ en Hongrie. 
On s'attardera quelques instants pour évoquer d'autres découvertes exceptionnelles et en lien avec l'émergence lente et complexe d'un travail des matières osseuses au Paléolithique. Le site allemand de Schöningen (13 II-4), dans un contexte daté autour de 300000 ans, a livré plusieurs longues pointes massives en bois végétal ${ }^{46}$ interprétées comme des armes de chasse (« lances, javelot»), et dont certaines présentent des traces de travail : de coupe et de raclage ${ }^{47}$. Cette dernière technique est très ponctuellement utilisée sur l'os au Paléolithique moyen pour seulement connaître un plein développement au cours du Paléolithique supérieur. Cette utilisation très précoce du bois végétal - aussi documentée à Bilzingsleben, en Allemagne et à Clacton-on-Sea, en Angleterre ${ }^{48}$ - interpelle quant à un vraisemblable transfert technique du bois végétal vers le bois animal; ces deux ressources organiques témoignant de fortes analogies structurelles $^{49}$. Si transfert il y a eu, ce dernier aura sans nul doute été occulté par des biais de conservation différentielle. Ceci aura favorisé la construction quelque peu artificielle, ou du moins exagérée, d'une maîtrise technique totalement aboutie des matières dures animales accompagnant la " révolution » cognitive et technique du Paléolithique supérieur, et implicitement l'arrivée de l'homme moderne en Europe. Les récentes études sur les débuts de l'Aurignacien en Europe et au Proche-Orient ${ }^{50}$, et plus largement sur les industries dites de transition ${ }^{51}$ montrent combien la réalité est plus complexe, en premier lieu car l'identité biologique des auteurs de ces traditions culturelles reste soit douteuse, soit inconnue ${ }^{52}$; deuxièmement car les débuts du Paléolithique supérieur ne s'accompagnent pas immédiatement d'un travail diversifié et systématique de l'ensemble des matières dures d'origine animale (cf. infra).

$\mathrm{Au}$ Paléolithique moyen, les témoins de l'exploitation des os à des fins techniques par les groupes néandertaliens se sont multipliés ces dernières années, tout en restant, pour l'heure, encore quantitativement faibles et relativement monotones (Fig. II, cahier couleur, $\mathbf{n}^{\text {os }} 3$ et 4). Sur les sites de cette époque, les productions se diversifient néanmoins, avec l'apparition de types nouveaux ${ }^{53}$. Les plus connus de ces outils sont les retouchoirs, même si quelques rares exemplaires sont signalés dans des contextes plus anciens, du Paléolithique inférieur ${ }^{54}$. Il s'agit d'éclats d'os bruts utilisés tels quels pour modifier les tranchants lithiques par percussion ${ }^{55}$. Ce sont leurs parties actives, présentes à la surface de l'os, sous forme de petites plages formées d'entailles et de stries, qui permettent de les identifier ${ }^{56}$. On connaît également quelques éclats retouchés qualifiés « de grattoirs, racloirs et denticulés ", en Italie sur le site de Fumane ${ }^{57}$, à Abric Romaní ${ }^{58}$ et à Axlor en Espagne ${ }^{59}$. Ce dernier site livre aussi quelques pièces intermédiaires sur éclats diaphysaires non façonnés ainsi que Karabi Tamchin en Ukraine ${ }^{60}$. Des poinçons et des " lissoirs » sont signalés sur le site allemand de Salzgitter-Lebensted ${ }^{61}$. Très récemment, des fragments de lissoirs, similaires à ceux du Paléolithique supérieur, mais datés de plus de 50000 ans, ont été découverts en Dordogne à Pech de l'Aze I et à l'abri Peyrony, dans des contextes stratigraphiques fiables. Ces outils témoignent d'une forte homogé- 
néité de forme - toutefois conditionnée par l'exploitation de tronçons de côtes de cervidés peu transformées (seule la partie active est façonnée $e^{62}$ ). Enfin signalons que, dans certains contextes moustériens méditerranéens italiens ou grecs, ce ne sont pas des éclats d'os qui sont travaillés par retouche, mais des coquillages marins, principalement Callistachione, pour faire des sortes de grattoirs/racloirs ${ }^{63}$.

Depuis quelques années, une recherche accrue de ces témoins discrets, difficilement discriminables des simples restes de boucherie, a pour corollaire une augmentation constante des corpus désormais disponibles, et tend à nuancer sensiblement le caractère épisodique de ce type d'exploitation technique pour les périodes anciennes. Certes, ces découvertes sont encore rares, et l'on ne retrouve pas la variété des techniques, des savoir-faire, des matières exploitées et des types qui caractérisent l'essentiel des industries osseuses du Paléolithique supérieur. Pour autant, les changements que l'on observe dessinent désormais une réalité beaucoup plus complexe que l'on ne l'aurait imaginée, il y a encore une décennie. Si des biais taphonomiques ont assurément contribué à minorer l'importance de ces productions osseuses anciennes, cet état de fait tient aussi aux débats très passionnés qu'elles ont suscités sur les capacités cognitives des Néandertaliens. Issus de contextes taphonomiques, stratigraphiques complexes, leur paternité néandertalienne fut souvent remise en question, considérant que ces outils étaient le fait direct (contaminations inter-couches...) ou indirect (échange, transmission de savoirs...) de l'homme moderne. En effet, ces découvertes concernent des occupations relativement récentes (autour de 40000 ans), et donc potentiellement contemporaines de l'arrivée de l'homme moderne en Europe. En ce sens, les récentes découvertes en Dordogne (cf. supra) démontrent que ce type d'exploitation technique est attesté en Europe bien avant l'arrivée d'Homo sapiens, tandis qu'en Afrique, dans le Middle Stone Age, si les exemples sont encore plus anciens, ils sont exclusivement rapportés à l'homme anatomiquement moderne.

\section{Le Paléolithique supérieur ancien : un moment d'innovations mais aussi de continuités avec le Paléolithique moyen}

En 1912 avec les subdivisions du Paléolithique supérieur, l'abbé Breuil est le premier préhistorien à se servir des outils en os comme " fossile directeur ", témoignant ainsi de la réalité et de la stabilité d'une industrie osseuse au Paléolithique supérieur. Pour autant, au Proto-Aurignacien, début du Paléolithique supérieur (42000 39900 cal. BP), les productions osseuses connues sont encore très lacunaires. Le travail de l'os est majoritaire, tandis que celui des autres matières osseuses reste exceptionnel; son exploitation révèle 
notamment d'importantes continuités avec les productions du Paléolithique moyen (prédominance de la percussion et d'un outillage sur éclats dont le taux de transformation est limité, etc.). Vers 39000 cal. BP, les données se multiplient suffisamment pour que l'on soit tenté de penser la rupture avec le Paléolithique moyen, non pas avec le ProtoAurignacien, mais seulement à partir de $1^{\prime}$ Aurignacien ancien ${ }^{64}$. Dès ce moment, l'exploitation de l'ensemble des matières dures animales est clairement attestée (os, ivoire, bois de cervidé, dent, coquillage). Elle se fait selon des techniques ou des procédés nouveaux (perforation par raclage circulaire, segmentation ou appointement par raclage en diabolo, par sciage, etc.), ou en gestation (abrasion, raclage linéaire, techniques d'éclatement) chez les groupes humains pré-modernes, et qui permettent aux fabricants de s'affranchir, si ce n'est complètement, au moins en grande partie, des contraintes volumétriques et structurelles des matériaux. Ces groupes aurignaciens inventent aussi les principales catégories d'objets utilitaires et symboliques qui persisteront (Fig. III, cahier couleur), sous des formes différentes, tout au long du Paléolithique supérieur (armatures de chasse, percuteurs, outils intermédiaires, bâtons percés, parures, statuettes, flûtes, etc.), ou qui initient de nouvelles formes dans les types préexistants connus en contexte européen (lissoirs, poinçons). Il semblerait que ce soit aussi à partir de l'Aurignacien ancien qu'apparaît une technologie cynégétique en matières osseuses; la dizaine de pointes de projectiles connues, à ce jour, dans des contextes proto-aurignaciens semble par plusieurs aspects discutables (fiabilité de la provenance stratigraphique, de l'attribution chronoculturelle, etc.); une récente révision critique $^{65} \mathrm{du}$ contexte chrono-stratigraphique d'apparition des pointes à base fendue en Europe tend à démontrer que ces productions ne sont véritablement visibles dans le registre archéologique qu'entre 38900 et 35700 cal. BP, ce qui coïncident avec l'évènement climatique froid d'Heinrich 4 (HS4) et l'éruption volcanique Campanian Ignimbrite au sud de $1^{\prime} I t a l i e^{66}$. Le passage entre le Proto-Aurignacien et l'Aurignacien ancien en Europe serait concomitant d'un changement majeur dans l'équipement de chasse, puisque les armatures lithiques, pour beaucoup des microlithes, coexistent désormais aux côtés de pointes en bois animal. Ces dernières seront venues sans doute se substituer au bois végétal dans la réalisation des armatures composites (ajout de microlithes sur un fût en matière organique), bien qu'il soit également possible que les pointes en bois de cervidé aient été utilisées seules, sans éléments lithiques ajoutés, à l'Aurignacien ancien ${ }^{67}$.

Plus largement, rien ne permet d'exclure que toutes les innovations techniques et conceptuelles que nous associons à l'émergence d'une véritable économie des matières dures animales au Paléolithique supérieur, ne soient, en réalité, connues et appliquées depuis des millénaires sur le bois végétal. Tout ceci reste bien entendu très spéculatif, mais il faut garder à l'esprit que l'absence ne signifie pas l'inexistence. Selon cette hypothèse, l'économie des matières dures animales, telle qu'elle se 
matérialise au début du Paléolithique supérieur s'inscrirait non pas dans un processus de rupture radicale avec ce qui précède au Paléolithique moyen, mais au contraire dans sa continuité : le travail des matières dures animales ayant pour héritage des millénaires d'expérience du travail du bois végétal (et de la pierre). La véritable rupture conceptuelle avec le Paléolithique moyen ne résiderait donc peut-être pas tant dans les techniques, les savoir-faire et les équipements associés au travail des matières dures animales, mais dans le fait de recourir désormais à ces nouveaux matériaux. La question essentielle serait alors : "pourquoi ce transfert du végétal aux matières dures animales »? Ce choix a-t-il pour moteur une restriction de l'accès aux ressources végétales, suite à des changements environnementaux majeurs ${ }^{68}$ ? En définitive, si le bond quantitatif et qualitatif exceptionnel qui se produit à partir de 39000 cal. BP dans le travail des matières dures animales est indubitablement concomitant de l'expansion de l'homme moderne en Europe, il est encore difficile d'affirmer qu'il lui est strictement consubstantiel tant que les données anthropologiques feront défaut, notamment pour les faciès de transition et le Proto-Aurignacien ${ }^{69}$. Plus spécifiquement, le cas du Châtelperronien d'Arcy-sur-Cure en Bourgogne (France), bien qu'unique en Europe $^{70}$ et dont l'intégrité des séries a été fortement débattue ${ }^{71}$, questionne sur l'origine peut-être plus ancienne de nombre d'innovations rapportées aux Aurignaciens. Si cet exemple ne fait pas consensus, les découvertes faites dans le Moustérien européen tendent à confirmer que les capacités cognitives des Néandertaliens ne peuvent plus être considérées comme un argument pertinent pour expliquer ce basculement qui s'opère sous l'action majoritaire (mais peut-être pas exclusive) des hommes anatomiquement modernes. N'oublions pas que nos connaissances sur ces périodes se renouvellent grandement ces dernières années, et que de nouvelles découvertes ne cessent de bousculer les modèles dominants. On signalera ainsi la persistance très tardive et inattendue en Russie, sur le site de Byzovaya, d'industries lithiques caractéristiques de la tradition moustérienne datées de plus de 8000 ans après la disparition théorique de Néandertal, soit il y a environ 28500 ans. La paternité biologique de ces industries reste cependant inconnue et interroge sur une possible perduration tardive des groupes de Néandertaliens à des latitudes exceptionnelles (près du cercle polaire arctique), ou sur une origine sapiens de ces vestiges ${ }^{72}$, ce qui là encore révolutionnerait totalement les schémas aujourd'hui connus, même si ces hypothèses ont aussi été discutées ${ }^{73}$.

Quoiqu'il en soit, le développement des traditions aurignaciennes s'accompagne de transformations profondes dans la relation homme/animal. L'animal n'est désormais plus seulement pourvoyeur de viande, de graisse (pour consommation et combustible), de tendons et de peaux, il devient aussi utile pour son squelette " interne » (os) et « externe » (bois et défenses). Cette imbrication très forte entre approvisionnement alimentaire et approvisionnement artisanal aura nécessairement eu des conséquences sur l'organisation des pratiques et des stratégies cynégétiques désormais déterminée par de nouveaux impératifs économiques ${ }^{74}$. 


\section{Les matières premières}

Les matières premières utilisées pour fabriquer l'industrie au début de Paléolithique supérieur sont variées. Ce qui est frappant, c'est l'exploitation de nouvelles matières comme l'ivoire et le bois animal (renne et cerf majoritairement). Leurs acquisitions ont en commun de pouvoir se faire par la chasse (bois de massacre ou défense de mammouth fraîche), ou indépendamment des circuits alimentaires (bois de chute ou récupération de défenses fraîches ou subfossiles sur des carcasses de mammouth). De plus pour le bois, sa collecte saisonnière varie suivant les espèces, l'âge et parfois le sexe des individus (pour le renne). Cette collecte requiert une connaissance des lieux d'approvisionnement et implique de fait une connaissance approfondie de l'éthologie des cervidés et de leur territoire ${ }^{75}$.

À partir de l'Aurignacien, on observe une plus grande rationalisation des choix en amont de la transformation technique de l'os, traduisant une anticipation accrue des besoins ${ }^{76}$; tendance qui semble s'affirmer nettement à partir du Gravettien (34 - 25000 cal. BP, pour la France). Ainsi, parallèlement à la récupération sélective d'éclats osseux comme supports d'outils futurs parmi les déchets culinaires, il est intéressant de noter, à titre d'exemple, une exploitation récurrente d'os longs, notamment de renne, soigneusement divisés dans leur longueur pour fabriquer des poinçons étroits et réguliers ${ }^{77}$. Pour que des os, riches en moelle, puissent être exploités de la sorte, il aura fallu les récupérer intacts. Une coopération étroite aura dès lors été nécessaire entre les dif- férents acteurs de la chaîne de traitement de la carcasse animale : du chasseur au fabricant, en passant par le boucher, afin d'anticiper et de coordonner les besoins alimentaires et techniques ${ }^{78}$. Une même personne pouvait aussi être investie de ces différents statuts.

\section{Des techniques adaptées aux matériaux fibreux, mais une prépondérance de la percussion}

Le choix et le traitement technique des différentes matières dures animales au Paléolithique supérieur reposent sur une connaissance des propriétés morphologiques, dimensionnelles et biomécaniques des matériaux, afin de répondre au mieux aux exigences fonctionnelles et éventuellement esthétiques du futur objet. Ces propriétés structurelles des matériaux sont directement fonction de la provenance anatomique et taxinomique des blocs de matières premières, ainsi que de l'âge, voire du sexe de l'animal pourvoyeur.

Les savoir-faire impliqués dans la transformation de ces ressources ont connu diverses évolutions d'une culture à l'autre, au cours du Paléolithique supérieur. Pour autant, nombre de tendances communes sont aussi observées sur une longue diachronie; ces " universaux techniques » sont fonction des limites objectives que la structure et l'état de fraîcheur des matériaux imposent (loi physique des matériaux) aux fabricants lors de l'exploitation technique ${ }^{79}:$ le rainurage, le sciage, le raclage, l'abrasion, la percussion (directe/indirecte; linéaire, diffuse, etc.) constituent à ce titre le fonds commun du travail des matières 
dures animales. Prises isolément, ces techniques sont peu informatives. En revanche, leur fréquence d'utilisation, tout autant que leur organisation au sein de chaînes opératoires, selon des gestes et des outils particuliers, déterminent des "schémas conceptuels ", guidés par un " $\operatorname{projet}^{80} »$, qui eux détiennent des clefs précieuses de caractérisation des différents groupes culturels qui ont vécu en Europe pendant près de 30000 ans, avant le début de l'Holocène.

Ainsi le travail des matières dures animales à l'Aurignacien se caractérise notamment par une utilisation encore très importante de la percussion diffuse pour la production d'éclats, la régularisation de supports ou la création de parties actives. Parallèlement, la percussion indirecte linéaire ou punctiforme - utilisée pour le fendage des os et des bois de cervidé - prend une place nouvelle et très importante dans les systèmes d'exploitation des blocs osseux. Cette exploitation longitudinale des bois de cervidé rend compte d'une particularité de ce matériau, à savoir son caractère fibreux, à l'image du bois végétal. Le fendage est utilisé pour diviser des tronçons de matière, afin d'obtenir des supports allongés (baguettes) utilisés pour la fabrication quasi exclusive des armatures de chasse ${ }^{81}$. Ce type de débitage agissant par division successive du bloc, il ne laisse pas ou très peu de déchets de débitage, nécessitant - pour être identifié - une lecture fine des discrets stigmates techniques encore conservés sur les supports bruts et les objets finis. D'autres travaux récents permettent d'envisager un possible débitage des bois de cervidé par percussion diffuse, à l'aide d'un galet et d'une enclume ${ }^{82}$.
Au Gravettien, c'est un rapport encore plus distancié aux contraintes de la matière qui s'instaure, avec l'invention d'une nouvelle méthode de débitage (l'extraction de baguette) et d'un nouveau procédé (le double rainurage longitudinal) permettant de s'extraire fortement des contraintes volumétriques et structurelles des blocs osseux (os, ivoire, et surtout bois de cervidé). En réalisant deux sillons longitudinaux parallèles ou convergents sur le bloc, et en positionnement exactement ces dernières et leur écartement, le fabricant peut prévoir, au millimètre près, la forme et la dimension du futur support. Ce dernier peut être reproduit à l'identique et en série, être large ou étroit selon l'objectif poursuivi. Si le « refend » aurignacien (partition par fendage) permet un contrôle plus accru de la forme et de la taille des baguettes débitées ${ }^{83}$, qu'on $^{\prime}$ ne l'aurait initialement envisagé ${ }^{84}$, et un débitage très rapide des supports, le taux de prédétermination en jeu dans cette méthode de débitage n'est cependant en rien comparable à celui qu'autorise l'extraction par double rainurage longitudinal. Sans compter, que ce dernier, s'il est correctement finalisé (rainurage profond et continu sur tout le pourtour $\mathrm{du}$ futur support), empêche quasiment tout accident lors du débitage. Les raisons de cette innovation technique sont des plus complexes à saisir, mais il apparaît clairement, au moins pour l'Europe occidentale, que cette nouvelle manière de concevoir le débitage est intimement liée à une matière première en particulier (le bois de cervidé), et est concomitante de changements profonds dans les armatures de chasse (forme et système $\mathrm{d}^{\prime}$ emmanchement ${ }^{85}$ ). 
L'équipement quotidien : des formes simples et des formes complexes?

S'il est une tendance relativement commune à l'ensemble des industries du Paléolithique supérieur, c'est bien l'utilisation majoritaire, parfois quasi-exclusive (Aurignacien), de l'os pour fabriquer les outils de transformation (poinçons, lissoirs, retouchoirs, etc.). Son accessibilité facile en faisait un matériau adapté aux impératifs économiques qui soustendent l'utilisation de ces outils; leur faible taux de transformation fréquent et leur durée de vie parfois très courte, auront sans doute favorisé l'utilisation d'un matériau aisément renouvelable ${ }^{86}$. Mais ce qui frappe le plus, c'est l'hétérogénéité de ces productions sur os : certaines sont faites sur de "simples" éclats, d'autres mettent à profit la forme naturelle de l'os - directement utilisée sans transformation aucune - tandis que d'autres enfin font intervenir une transformation, parfois totale, de l'élément osseux. Ces différentes modalités d'exploitation coexistent fréquemment au sein d'une même période culturelle, voire d'un même site. L'adjonction de décors - le plus souvent géométrique est en outre fréquente sur ces outils, y compris sur ceux peu ou non façonnés. L'os est aussi utilisé pour la fabrication de contenants, de parure tandis que son utilisation comme support de fabrication des pointes de projectiles reste encore assez peu documentée.

Le bois de cervidé est (à partir du Gravettien) fréquemment utilisé pour des productions en lien avec la transformation d'autres matériaux (coin et ciseau, bâtons percés, compresseurs, etc.), néanmoins, depuis l'Aurignacien, il reste le matériau privilégié pour la confection des armatures de projectiles ${ }^{87}$, tout du moins en Europe occidentale.

Si quelques percuteurs en bois de cervidé, et exceptionnellement en ivoire, sont connus à $1^{\prime}$ Aurignacien ${ }^{88}$ et au Gravettien ${ }^{89}$, ces outils, utilisés comme des marteaux afin de détacher des éclats d'un bloc, sont surtout identifiés en contexte solutréen. Le premier à avoir été identifié, en 1972 par François Bordes et Jacques Tixier $^{90}$, provient du site de Laugerie-Haute Ouest. Depuis, les découvertes se sont multipliées jusqu'à faire du Solutréen la période la plus riche en percuteurs sur partie basilaire de bois de cervidé. Toutefois, cela ne représente jamais qu'une petite vingtaine d'exemplaires, essentiellement répartie dans le sud-ouest de la France et le nord de $1^{\prime}$ Espagne ${ }^{91}$. C'est très peu, surtout lorsque l'on considère que leur utilisation était très largement répandue ce dont témoignent indirectement les techniques de taille et de retouche du matériel lithique. La plupart des percuteurs solutréens sont assez graciles, mais denses. Selon une échelle de poids qui détermine leur fonction précise, on peut les ranger dans des tranches adaptées à la retouche (de grattoir notamment), à la finition des feuilles de laurier solutréennes et au plein débitage de lamelles (150 à 300 gr. exemplaire de Laugerie-Haute) ou qui les rendent compatibles avec le façonnage des petites et moyennes feuilles de laurier (300 à 500 gr.) (2) $^{92}$ Il existe du reste une étroite relation entre les percuteurs tendres et les industries à pointes foliacées, solutréennes ou non. Pour le Solutréen, que ce soit à Laugerie-Haute Ouest, à Combe-Saunière (Dordogne), à 
l'Arbreda (Espagne) ou à Solutré (Saône et Loire), il est systématiquement fait état de la présence concomitante de feuilles à retouches bifaciales fines et de percuteurs en bois de renne. Pour l'exemplaire de Laugerie-Haute cela dépasse même la simple coïncidence puisque de petites esquilles de silex calcédonieux ${ }^{93}$ ont été identifiées figées dans la partie active du percuteur $^{94}$.

Un autre outil solutréen est aussi clairement associé au travail de la pierre, mais par pression, il s'agit du compresseur. Ce sont des objets sur baguette de bois de cervidé, principalement de renne, et plus rarement en os de gros ou moyens mammifères. Ils présentent tous les mêmes stigmates caractéristiques d'enlèvements de matière et de déformation dans leur partie active ${ }^{95}$. Identifiés dès 1938 par Denis et Elie Peyrony, ces pièces sont les oubliées de l'industrie osseuse solutréenne alors qu'ils constituent probablement aujourd'hui une des principales catégories d'outils ou tout du moins, la plus caractéristique pour cette période ${ }^{96}$.

Le reste de l'équipement domestique est représenté par le fonds commun classique des équipements paléolithiques ${ }^{97}$ : os à impressions (enclume, retouchoir) liés à diverses activités de transformation et de fabrication, bâtons percés, dont la fonction reste encore énigmatique, lissoirs et poinçons associés au travail des matières souples animales ou végétales (peaux, tiges, feuilles...); armatures de sagaies de types variés pour la chasse, petits éléments bipointes aux deux fonctions possibles : cynégétique (fixés au bout d'une hampe comme les armatures de sagaies) et halieutique (hameçons droits). Néanmoins, les Solutréens y ont apposé leur particularité en aménageant, comme sur une grande part des objets de parure, des crans ou des stries courtes parallèles et perpendiculaires sur une proportion parfois très importante d'objets domestiques en os. Enfin, à la fin du Solutréen, en Europe occidentale, apparaissent deux inventions majeures : le propulseur ${ }^{98}$ et l'aiguille à chas ${ }^{99}$. Toutes deux vont connaître un développement considérable au cours des périodes suivantes voire perdurer sous la même forme jusqu'à nos jours ${ }^{100}$. L'invention du propulseur et celle de l'aiguille à chas ne sont attestées alors que par quelques pièces. Pour les aiguilles à chas, elles sont présentes sous une forme très sporadique dans deux foyers régionaux distincts : la Vallée de la Vézère et le Nord-ouest de l'Espagne et, pour les propulseurs, uniquement en Dordogne; le plus ancien exemplaire connu provient du site périgourdin de CombeSaunière I, quoique son attribution au Solutréen soit discutée. Un autre exemple possiblement solutréen a été découvert à la grotte du Placard, en Charente ${ }^{101}$. En l'état des connaissances, les plus anciens propulseurs issus de contextes fiables appartiennent aux premières phases du Magdalénien moyen, antérieur à 18000 BP dans le sud-ouest français ${ }^{102}$. L'aiguille à chas est en revanche connue plus tôt en Europe orientale. Plusieurs sites russes de la culture de KostienkiAvdeevo, datés de 28000 - 25000 cal. BP, livrent ce type d'outil ${ }^{103}$. 


\section{Le Paléolithique supérieur récent : des conceptions du travail des matières dures animales variées...}

\section{Badegoulien et Magdalénien ancien}

En l'état actuel des recherches, la distinction culturelle entre les groupes du Magdalénien ancien et ceux du Badegoulien n'est pas clairement établie. Une différence fondamentale semble pourtant les distinguer dans leur façon de travailler les matières osseuses. En effet, si le débitage par extraction est bien présent au Magdalénien ancien, il semble avoir été remplacé au Badegoulien par un débitage original qui occupe une place prépondérante; ce dernier consiste à éliminer progressivement de la matière $\mathrm{du}$ bloc sous forme de fragments ou d'éclats pour en dégager un support allongé (type éclat baguettaire), par des procédés d'éclatement associant percussion diffuse et percussion tranchante. Les nombreux autres éclats produits par ce type de débitage sont généralement considérés comme des déchets, mais certains pourraient aussi avoir servi de supports d'outils (bruts ou façonnés ${ }^{104}$ ). Ce débitage est indifféremment appliqué au bois de renne et à l'os et conduit, semble-t-il, à produire les mêmes types d'objets que ceux issus d'un débitage par extraction : des pointes de projectile et des outils biseautés dans le premier cas; des aiguilles et des alênes dans le second (Fig. IV, cahier couleur). Toutefois, en raison du nombre limité de séries connues et étudiées, la composition de l'équipement des groupes relatifs à l'un ou l'autre reste mal connue ${ }^{105}$. Parmi les objets et instruments liés aux activités de transformation et de fabrication, on note la présence en nombre des outils intermédiaires à biseau distal bifacial sur baguette et le développement net des aiguilles à chas ${ }^{106}$. Ces pièces sont associées au fonds commun des groupes du Paléolithique supérieur: poinçons divers, bâtons percés à branches courtes ou sans branches, lissoirs communs, etc. Parmi les objets et instruments liés à la chasse et à la pêche, on retrouve des armatures de projectile bipointes, des baguettes demi-rondes et des propulseurs; ces derniers n'étant clairement attestés qu'au Magdalénien moyen ancien ${ }^{107}$ ).

Magdalénien moyen et récent : l'apogée des productions en matières osseuses

À partir de 19000 cal. BP, les groupes magdaléniens font preuve d'une grande maîtrise technique, que ce soit pour débiter les supports ou les mettre en forme et obtenir des objets finis très standardisés, témoignant d'une forte structuration de l'économie. Leur équipement est fabriqué sur des supports obtenus à partir de deux schémas prédominants de transformation. Le premier implique un débitage par extraction qui vise à obtenir des supports de forme prédéterminée type « baguette » ou « disque ». Ils sont aménagés ensuite, dans le premier cas, en sagaies, harpons, baguettes demirondes, parfois en propulseurs, aiguilles, poinçons, alênes, lissoirs, etc., et, dans le second, en rondelles, pendeloques, ellipses. Le second schéma implique un 
débitage par segmentation conduisant à produire des tronçons qui, à partir de bois de renne de petit ou moyen module, permettront notamment d'obtenir des bâtons percés, des propulseurs et des percuteurs. Dans les deux cas, un grand soin est apporté à la régularisation finale des surfaces, l'installation de décor via des gravures ou des sculptures parfois en ronde-bosse, l'application d'une substance colorante et/ou protectrice.

\section{Une forte standardisation des moyens et des principes de fabrication}

Dès la phase moyenne, les groupes magdaléniens exploitent toute la finesse de réalisation qu'autorise l'emploi du procédé d'extraction par double rainurage, créant des types distincts de baguettes, parfois sur un même bloc osseux (os ou bois), en attribuant à chacun une destination fonctionnelle précise $^{108}$ : la longueur et la section du support extrait est ainsi en adéquation avec la morphologie du futur objet. Les formes sont si normées qu'il est possible de créer des catégories typo-techniques à partir des supports, indice d'une forte standardisation (baguette en tasseau, en boudin...). Cette dernière se retrouve aussi dans la morphologie et les modules de bois sélectionnés pour la fabrication, alors qu'une telle homogénéité morphométrique n'existe pas dans les troupeaux de rennes, les bois ont donc été soigneusement choisis.

$\mathrm{Au}$ cours de la phase récente du Magdalénien, ces séries s'accroissent quantitativement grâce à la haute productivité des débitages qui permettent d'obtenir un grand nombre d'objets à partir d'un seul bloc de matière première. Dans le sud-ouest de la France, le débitage par extraction multiple périphérique connaît alors son apogée. Ce débitage, réalisé par un rainurage longitudinal parallèle, associé à un détachement transversal par percussion diffuse souvent guidé par l'aménagement d'une gorge, est indifféremment appliqué à l'os long ou aux perches de bois de cervidé en suivant exactement les mêmes principes. Dans le nord de la France, en particulier dans le Bassin parisien sur les sites de Pincevent, Verberie et Étiolles, une autre variante du débitage par extraction a été préférée ${ }^{109}$. Il s'agit de la production d'une grande baguette unique tout le long de la face antérieure de la perche de bois de renne de gros module. Celle-ci sera ensuite sectionnée transversalement en trois à quatre baguettes plus petites, utilisées pour produire des pointes de projectile ou des outils intermédiaires biseautés.

\section{Un développement en nombre et en formes de l'équipement}

$\mathrm{Au}$ Magdalénien moyen, l'équipement de chasse et de pêche est constitué notamment de très nombreuses baguettes demi-rondes avec parfois un décor géométrique, spiralé ou à tubérosité ${ }^{110}$; des propulseurs de types variés et dont certains ont été très richement décorés avec des sculptures en rondebosse; des armatures de sagaie principalement à biseau unifacial ou bifacial qui connaissent un fort développement dans tout le bassin d'Aquitaine, dans les Pyrénées et en Espagne Cantabrique, et enfin aussi des bipointes. Ces armatures sont dans certaines régions accompa- 
gnées de nouvelles formes, présentes en quantité dans quelques sites pyrénéens pour les armatures de sagaie à base fourchue ou les sites du seuil du Poitou, pour les sagaies de Lussac-Angles ${ }^{111}$.

Parmi les outils du fonds commun on trouve continuellement des bâtons percés, des percuteurs sur partie basilaire de bois de renne, des outils intermédiaires, notamment sur baguette à biseau bifacial, des os à impressions et des objets appointés, type poinçons.

$\mathrm{Au}$ Magdalénien récent, à partir de 17000 cal. BP, le processus de multiplication et d'apparition de nouveaux types d'objets se poursuit. Parmi l'équipement lié aux activités de chasse et de pêche, de nouveaux objets barbelés apparaissent dont les harpons ${ }^{112}$ à tête détachable et les foënes et on trouve aussi la plupart des préhampes connues, c'est-à-dire des pièces intermédiaires entre la hampe et la pointe de projectile. Cet équipement de chasse est accompagné de composants plus classiques comme les éléments droits à double pointe, les armatures de sagaie à biseau uni ou bifacial, à base fourchue, les bipointes, les baguettes demi-rondes et les propulseurs qui n'ont plus de sculptures en ronde-bosse. Dans le groupe du fonds commun, cette période connaît quelques éléments remarquables comme la multiplication des zones d'extension des aiguilles à chas (Provence, Espagne du sud, Angleterre, Belgique, Allemagne, Pologne, Autriche, Tchécoslovaquie, Hongrie); des rares bâtons percés de type multiforé et des lissoirs de type déjeté reconnu pour l'instant seulement à La Vache. Les outils intermédiaires à biseau uni ou bifacial, les os à impression les tubes (notamment avec épiphyses perforées et des décors figuratifs très particuliers), enfin les poinçons complètent cette liste. À la fin du Magdalénien, apparaît le premier objet en forme de cuiller à Gourdan ${ }^{113}$, mais ce type va seulement se développer à partir du Néolithique ancien avec des sociétés à économie de production.

\section{Une gestion saisonnière de la production des équipements}

Matière première d'excellence des Magdaléniens, le bois de renne prédomine largement sur les autres matières osseuses. Ce sont essentiellement des bois de mue (tombés naturellement) qui ont été utilisés et qui, selon leurs modules $^{114}$ et leurs caractéristiques, sont attribuables à des rennes mâles adultes (gros module), à de jeunes mâles ou des femelles adultes (petit et moyen modules selon le développement des bois). On peut, en simplifiant, distinguer deux principales périodes d'acquisition de la matière première - fin de l'automne / début de l'hiver (bois de gros module) et le printemps (bois de petit module). Certains modules semblent associés à certains types de production et peut-être à une saison. Ainsi en hiver, les Magdaléniens fabriquent une grande partie de leur équipement cynégétique en bois de renne sur des gros modules (pointes de projectile par exemple) et de l'outillage associé au travail de ce matériau (outils biseautés); au printemps, ils l'ont complété avec d'autres instruments de chasse sur des bois généralement de petit module (principalement des propulseurs ${ }^{15}$ ) ou d'autres objets (des bâtons percés ou certains types de ciseaux). Cette dichotomie est bien sûr très schématique; la réalité est toujours 
plus complexe et, de fait, il a été observé que les bois de moyen module sont souvent utilisés pour compenser la production majoritaire de la saison ${ }^{116}$. Pour autant, au cycle saisonnier de production s'associerait un cycle de consommation différée, qui a pu s'étendre sur une ou plusieurs années (comme les pointes) voire des décennies (propulseurs, bâtons percés, percuteurs), selon les objets. Chaque année, les groupes n'auraient eu alors qu'à remplacer une partie de l'ancienne panoplie, s'assurant de la sorte d'avoir toujours à disposition les pièces essentielles de leur équipement.

À la fin du Magdalénien, un changement s'annonce : si la continuité dans les principes conceptuels et pratiques du débitage du bois de renne au Magdalénien récent est évidente, en revanche, il semble que les débitages deviennent moins productifs en termes de supports comme de variété d'objets finis ${ }^{117}$. Parallèlement, se développe l'exploitation du bois de cerf qui, comme souvent sur ce type de bloc, associe à la fois un débitage par extraction et un débitage par tronçonnage : le premier conduit à la production d'un type particulier de bâton percé aménagé sur une large baguette; le second conduit à la production d'objets intermédiaires biseautés, de type coin.

\section{D'autres temps, d'autres habitudes, d'autres équipements}

L'image d'Épinal de l'équipement post-magdalénien tient à son apparente pauvreté, à la fois quantitative et qualitative. En effet, l'industrie osseuse des groupes aziliens, comparée à l'exceptionnelle richesse de celle des Magdaléniens, est pauvre, quel que soit le contexte géographique (Fig. IV, cahier couleur, $\mathbf{n}^{\text {os }} 7$ à 9). Comment l'expliquer? D'importants changements interviennent à la fin du Paléolithique supérieur dans l'ensemble des domaines de la culture matérielle et symbolique. Est-ce que l'environnement plus forestier aurait amené, lors des chasses, une préférence pour des pointes de projectiles lithiques, légères et rapides à remplacer, aux dépens des pointes lisses en matières osseuses privilégiées par les groupes antérieurs ${ }^{118}$ ? De fait, les crochets de propulseurs en matières osseuses tendent à disparaître dès le Magdalénien supérieur et disparaissent totalement, dans le contexte européen, à partir de l'Azilien. Simultanément, l'armement en matières dures d'origine animale est réduit aux pointes barbelées, et surtout aux harpons à tête détachable. La forme et la matière première de ces derniers évoluent également : ils sont fabriqués en bois de cerf et deviennent plus plats et moins élaborés (en particulier au niveau des barbelures et du système de rétention). Il est vrai que les données sont quantitativement faibles pour cette période. Un récent recensement bibliographique fait état d'un peu plus de 600 pièces aziliennes identifiées sur 72 gisements en France et en Espagne ${ }^{119}$. 
L'industrie est surtout caractérisée par des objets; quant aux restes de fabrication, ils sont rarement identifiés dans les sites. Les quelques indices, tout du moins pour le bois de cerf, indiquent la production de baguettes par double rainurage parallèle longitudinal (La Tourasse, Pont d'Ambon, peut-être Troubat), ou par partition par fendage à Pégourié ${ }^{120}$. La plupart des outils en os sont sur éclats de diaphyse, sans que l'on puisse toutefois distinguer, dans cette fracturation, l'intention technique de l'objectif alimentaire. L'équipement domestique se caractérise par une simplification des formes. Les bâtons percés et les baguettes demi-rondes disparaissent, ainsi que toute une panoplie d'objets d'art mobilier sur support divers. Ce sont essentiellement des objets appointés, type poinçons sur supports non normés (sauf pour l'os vestigial) et quelques rares lissoirs sur côte. Un nouvel instrument, peut-être de pêche, apparaît de façon sporadique, l'hameçon courbe, à moins qu'il ne s'agisse d'un simple crochet à suspendre ${ }^{121}$.

\section{Conclusion}

Pendant la Préhistoire, en Europe, les matières dures $d$ 'origine animale ont été utilisées comme matière première. $\mathrm{Au}$ début, au Paléolithique ancien et moyen, l'os est choisi ponctuellement pour fabriquer des outils peu modifiés, comme certains bifaces ou retouchoirs. Mais c'est surtout à partir du Paléolithique supérieur (vers 40000 BP) que ces matières ont été largement choisies par les sociétés de chasseurs-cueilleurs pour produire une panoplie variée, à la fois sur os et bois animal : des armes et instruments de chasse (pointes de projectiles, propulseurs...), outils quotidiens (bâtons percés, poinçons, lissoirs, aiguilles, coins et ciseaux...), objets de parure (perles, pendeloques et bracelets) et objets symboliques (statuettes et autres objets d'art mobilier). Le comportement des derniers chasseurs-cueilleurs vis-à-vis de ces matériaux évolue, une importance moindre est accordée à l'industrie osseuse et des outils de

formes plus simples, mais certainement aussi efficaces sont alors produits. De nouvelles catégories apparaissent, liées au développement de l'artisanat des matières végétales, dont le bois.

En définitive, l'exploitation des matières dures d'origine animale au cours du Paléolithique est à l'image de l'évolution de la lignée humaine : buissonnante et complexe. Elle est jalonnée de moments de stabilité au sein des systèmes techniques, que nous pouvons interpréter comme des moments de stabilité sociale et économique, mais aussi de changements progressifs ou rapides. Ces moments où le système technique se modifie, que ce soit sous l'impulsion de facteurs internes au groupe (modification de la structure démographique, des relations sociales, etc.), ou de facteurs externes (changements environnementaux, climatiques, conflits, arrivée de nouvelles populations...), peuvent se traduire par une évolution qualitative 
et/ou quantitative au sein des ensembles archéologiques étudiés. Des moments de changements brusques, avec interruption d'un système technique et apparition d'un nouveau, sont aussi à l'œuvre tout au long de la Préhistoire récente. Ces transformations radicales traduisent de véritables ruptures conceptuelles, techniques et culturelles. L'identification de ces rythmes évolutifs et des mécanismes socio-culturels impliqués est actuellement un enjeu structurant de la technologie osseuse, et de la recherche préhistorique ${ }^{122}$ en général. L'une des difficultés en la matière étant de s'assurer que les ruptures que nous percevons reflètent bel et bien une réalité préhistorique et non un découpage artificiel de préhistorien. Des jalons de compréhension intermédiaires nous font sans doute fréquemment défaut. Dès lors, il est essentiel de s'assurer que nous ne sommes pas en présence de continuités historiques artificiellement morcelées par des biais de conservation différentielle ou par l'incapacité de nos méthodes de datation à saisir l'écoulement du temps court ${ }^{123}$. Une autre difficulté réside dans nos résolutions d'analyse, certes de plus en plus perfectionnées et précises, mais parfois si spécialisées, qu'elles en deviennent restrictives dans leurs ambitions paléohistoriques. Le fait technique doit donc sans cesse être repensé dans sa dimension anthropologique.

Le potentiel informatif des industries en matières dures d'origine animale qu'il reste à étudier est, à ce titre, considérable et constitue un enjeu scientifique d'autant plus stimulant, que les nouvelles découvertes en la matière ne cessent de bousculer nos certitudes, voire de les renouveler radicalement. Ces productions, plus que d'autres sans doute, sont à l'interface de différentes sphères de la culture matérielle, idéelle et de l'économie de subsistance. Elles offrent un trait d'union tangible entre l'exploitation alimentaire et technique des ressources animales, mais aussi avec le travail de la pierre et la finalité des productions associées, tout autant qu'avec l'univers des croyances et des symboles (art mobilier, objets osseux décorés, parures, instruments de musique) des chasseurscueilleurs du Paléolithique. Elles nous apportent des éclairages singuliers sur le rapport de l'homme à son environnement terrestre, mais aussi maritime (travail des coquillages ${ }^{124}$ et plus rarement d'ossements de mammifères marins ${ }^{125}$.)

s'adressent aussi à Robert Begouën, Céline Bemilli, Miguel Biard, Giovanni Boschian,CaroleFritz,Stéphan Hinguant, Shannon McPherron, Caroline Peschaux, Jean-Marc Pétillon, Marie Soressi, JoséMiguel Tejero et Gilles Tosello. Nous remercions aussi Peggy Jacquement, 
Bernard Nicolas pour leur aide toujours essentielle sur les collections du MNPE, Mathieu Leroyer pour ses remarques avisées et enfin, Pierre Cattelain, pour sa relecture du présent article.

\section{Notes}

1. Gustave CHAuvet, Os, ivoires et bois de renne ouvrés de la Charente. Hypothèses palethnographiques, Bulletin et mémoires de la Société archéologique et historique de la Charente, t. 1, 1910, p. 1-184.

2. Léon HENRI-MARTIN, « La percussion osseuse et les esquilles qui en dérivent. Expérimentation », Bulletin de la Société préhistorique de France, vol. 5, $\mathrm{n}^{\circ} 7,1910$, p. 299-304.

3. Sergey A. Semenov, Prehistoric technology, Londres, Moonraker Press, 1964.

4. Aline Averboun, Technologie de la matière osseuse travaillée et implications palethnologiques. L'exemple des chaînes d'exploitation du bois de cervidé chez les Magdaléniens des Pyrénées, thèse de doctorat, université de Paris 1 Panthéon-Sorbonne, Paris, 2000.

5. Marc Gröenen, Pour une histoire de la Préhistoire. Le Paléolithique, Grenoble, Jérôme Millon, L'Homme des Origines, 1994.

6. Boris VAlentin, Jalons pour une Paléohistoire des derniers chasseurs (XIVe-VI millénaires avant J.-C.), Cahiers archéologiques de Paris 1, Publications de la Sorbonne, 2008.

7. Nejma Goutas et Jose-Miguel Tejero, " Osseous Technology. Another way to catch chronological, economic and social aspects of hunter-gatherers Palaeolithic groups ", in Aline Averbouh, José-Miguel Tejero, Nejma Goutas et Marianne Christensen (eds.), Innovation in the production and use of equipment in hard animal materials: origins and consequences in prehistoric societies from Palaeolithic to Mesolithic, XVII Congress of the UISPP (Burgos, Spain), 1-7 September 2014, Quaternary international, Proceedings of the session B24, 2016, p. 79-89.

8. Ibid., p 80 .

9. Boris VALENTIN, «Typologie vs typologie (Sic!). Comment la technologie contribue à raffiner la typologie des armatures lithiques ", in Thierry Aubry, Francisco Almeida, Anna-Cristina Araújo, Marc Tiffagom (dir.), Space and Time: Which Diachronies, Which Synchronies, Which Scales? Typology vs Technology, Proceedings of the
XV World Congress of the International Union for Prehistoric and Protohistoric Sciences, 21, Sections C64 and C65, p. 189-196, 2008.

10. Aline Averbour et Noëlle Provenzano, «Propositions pour une terminologie du travail préhistorique des matières osseuses : I. Les techniques ", Préhistoire Anthropologie Méditerranéennes, 7, 1999, p. 5-25; Aline Averbouh, op. cit., 2000.

11. D'après André Cheynier in Lucette Mons, Fiche "sagaie à base raccourcie », in Henriette CAmps-Fabrer (dir.), Fiches typologiques de l'industrie osseuse préhistorique. Cahier 1 : Sagaies, Aix-enProvence, édition université de Provence, 1988, p. 1-9.

12. Jacques Allain, «Réflexion sur la chronologie du Magdalénien ", Bulletin de la Société Préhistorique Française, 55, 9, 1958, p. 539-545.

13. François-Xavier Chauvière et André Rigaud, "Les "sagaies" à "base raccourcie" ou les avatars de la typologie : du technique au "non-fonctionnel" dans le Magdalénien à navettes de la Garenne (Saint-Marcel, Indre) ", in Véronique DUJARDIN (dir.), Industrie osseuse et parures du Solutréen au Magdalénien en Europe, table ronde sur le Paléolithique supérieur récent, Angoulême, 28-30 mars 2003, Paris, édition Société Préhistorique Française, 2005, p. 233-242.

14. Nejma Goutas, Caractérisation et évolution $d u$ Gravettien en France par l'approche techno-économique des industries en matières dures animales (étude de six gisements du sud-ouest), thèse de doctorat, université Paris 1 Panthéon-Sorbonne, Paris, 2004.

15. Élise TARTAR, De l'os à l'outil. Caractérisation technique, économique et sociale de l'utilisation de l'os à l'Aurignacien ancien. Étude de trois sites : l'Abri Castanet (secteurs nord et sud), Brassempouy (Grotte des Hyènes et Abri Dubalen) et Gatzarria, thèse de doctorat, université de Paris 1 Panthéon-Sorbonne, 2009.

16. Anne VINCENT, « L'os comme artefact au Paléolithique moyen : principe d'étude et premiers résultats ", in Lewis R. BINFORD et Jean-Philippe Rigaud (dir.), L'homme de Néandertal, 4, La technique, Belgique, ERAUL, 1988, p. 185-196.

17. Élise TARTAR, « L'analyse techno-fonctionnelle de l'industrie en matières osseuses dite 'peu élaborée'. L'exemple des pièces intermédiaires en os de l'Aurignacien ancien de la Grotte des Hyènes (Brassempouy, Landes) », in Aline AverbouH et Marianne CHRIstensen (dir.), Transformation et utilisation préhistoriques des matières osseuses. Actualité des recherches universitaires en France 20002004, Aix-en-Provence, Préhistoire Anthropologie Méditerranéennes, 2003, p. 139-146.

18. Ibid., p. 139; Emiliano Aguirre et Marylène Patou (dir.), Outillage peu élaboré en os et bois de cer- 
vidé I, coll. " Artefacts », 1, Treignes, édition du Centre d'études et de documentation archéologiques, 1985.

19. Marcel Moussette, « Sens et contre-sens : l'étude de la culture matérielle au Québec ", Canadian Folklore canadien, $\mathrm{n}^{\circ} 4,1984$, p. 11-15.

20. C'est-à-dire, la connaissance des moyens qui permettent l'accomplissement d'une tâche dans des conditions d'exactitude et de précision, voir notamment : Marie-Louise InIZAN, Michèle Reduron, Jacques Tixier et Hélène Roche, Préhistoire de la pierre taillée 4, Paris, C.R.E.P, 1995.

21. André Leroi-Gourhan, Évolution et technique I. L'homme et la matière, Paris, Albin Michel, 1971 [1943] ; André-Georges Haudricourt, "L'origine des techniques ", Courrier rationaliste, $\mathrm{n}^{\circ} 12,1965$, p. 32-36; Sylvie Ploux, Approche archéologique de la variabilité des comportements techniques individuels : les tailleurs de l'unité 27-M89 de Pincevent, thèse de doctorat, université de Paris 10, 1989; Nicole Pigeot, Entre Nature et Culture. Valeur heuristique de la technologie lithique par des approches systémiques et cognitives, thèse $\mathrm{d}$ 'habilitation à diriger les recherches, université de Paris 1 Panthéon-Sorbonne, Paris, 1991; Jacques Pelegrin, "Aspects de démarche expérimentale en technologie lithique », in 25 ans d'études technologiques en Préhistoire. Bilan et perspectives. XI rencontres internationales d'Archéologie et d'Histoire d'Antibes. 18-20 octobre 1990, Juan-les-Pins, APDCA, 1991, p. 57-63; Catherine PERLÈs, "Quand diffusion ne veut pas dire interaction », Congrés Internacional Xarxes al Neolític - Neolithic Networks Rubricatum (Revista del Museu de Gavà, num. spéc. n 5, 2012, p. 585-589).

22. Voir par exemple : Aline Averboun, Robert Begoüen et Jean Clottes, "Technique et économie du travail du bois de cervidé chez les Magdaléniens d'Enlène (Montesquieu-Avantès, Ariège) : vers l'identification d'un cycle saisonnier de production ", in Michèle Julien (dir.), Préhistoire d'os. Recueil d'études sur l'industrie osseuse préhistorique offert à Henriette CampsFabrer, Aix-en-Provence, Publications de l'université de Provence, 1999, p. 289-318; Marianne Christensen et Boris Valentin, "Armatures de projectiles et outils : de la production à l'abandon ", in Nicole Pigeot (dir.), Derniers Magdaléniens d'Étiolles : perspectives culturelles et paléohistoriques, Paris, éd. CNRS, 2004, p. 107-160; Jessica Lacarrière, Nejma Goutas, Christian Normand et Aurélien Simonet, « Vers une redéfinition des occupations gravettiennes de la grotte d'Isturitz (Pyrénées-Atlantiques, France) : révision critique des collections anciennes par l'approche intégrée des données lithiques, fauniques et de l'industrie osseuse ", in Nejma Goutas, Laurent Klaric, Damien Pesesse et Patricia Guillermin (dir.), À la recherche des identités gravettiennes. Actualités, questionnements et perspectives, Actes de la table ronde internationale du 6-8 octobre 2008, Aix-en-Provence, Mémoire LIII de la Société préhistorique française, 2011, p. 67-83; José-Miguel Tejero, Marianne Christensen et Pierre Bodu, "Red deer antler technology and early modern humans in southeast Europe : an experimental study ", Journal of Archaeological Science, vol. 39, $\mathrm{n}^{\circ}$ 2, 2012, p. 332-346; Jean-Marc Petillon et Sylvain DuCAsse, "From flakes to grooves: a technical shift in antler working during the Last Glacial Maximum in southwest France ", Journal of Human Evolution, vol. 62, n 4, 2012, p. 435-465; Élise TARTAR, Nicolas Teyssandier, François Bon et Despina Liolios, "Equipement de chasse, équipement domestique : une distinction efficace? Réflexion sur la notion d'investissement technique dans les industries aurignaciennes ", in Laurence Astruc, François BoN, Vanessa LÉA, Pierre-Yves Milcent et Sylvie Philibert (dir.), Normes techniques et pratiques sociales de la simplicité des outillages pré- et protohistoriques, $\mathrm{XXVI}$ rencontres internationales d'archéologie et d'histoire d'Antibes, Antibes, APDCA, 2006, p. 107-117.

23. André Leroi-Gourhan, Le Geste et la parole. II. la mémoire et les rythmes, Paris, Albin Michel, coll. Sciences d'aujourd'hui, 1964-1965; Jean GuILAINE, " La diffusion de l'agriculture en Europe une hypothèse arythmique ", Zephyrus, vol. 53-54, nº 2000-2001, 1998, p. 267-272.

24. Henri Breuil, " Le feu et l'industrie de pierre et d'os dans le gisement du Sinanthropus à Chou-Kou-Tien ", L'Anthropologie, no 42, 1932, p. 1-77.

25. Joseph KI-Zerbo, Méthodologie et Préhistoire africaine, Histoire générale de l'Afrique, vol. 1, Unesco, International Scientific Committee for the Drafting of a General History of Africa, 1997.

26. Louis LegUAY, "Les procédés pour la gravure et la sculpture des os avec le silex », Bulletin de la Société d'Anthropologie, vol. 12, n 2, 1877, p. 280-296.

27. Léon Henri-Martin, " La percussion osseuse et les esquilles qui en dérivent. Expérimentation », op. cit., 1910.

28. Luis SirET, «Emploi de l'os dans la retouche des silex moustériens ", Bulletin de la Société préhistorique de France, vol. 22, n 5, 1925, p. 208-210.

29. Francesco d'Errico et Paola Villa, « Holes and grooves : the contribution of microscopy and taphonomy to the problem of art origins », Journal of Human Evolution, vol. 33, nº 1, 1997, p. 1-31.

30. Francesco d'ERrico et Paola Villa, « Nouvelle analyse des os gravés et perforés du 
Paléolithique inférieur et moyen. Implications pour l'origine de la pensée symbolique ", Paleo, 1998, p. 265-285.

31. Francesco d'Errico et Paola Villa, « Bone and ivory points in the Lower and Middle Paleolithic of Europe », Journal of Human Evolution, vol. 41, no 2, 2001, p. 69-112.

32. Francesco d'ERrico et Paola Villa, " Nouvelle analyse des os gravés ", op. cit., 1998, p. 265-285.

33. Anne Vincent, L'outillage osseux au Paléolithique moyen : une nouvelle approche, thèse de doctorat, université de Paris 10, 1993.

34. Raymond Lantier et Henri Breuil, Les hommes de la pierre ancienne (Paléolithique et Mésolithique), Paris, Bibliothèque scientifique, Payot, 1959.

35. Henri Breuil, " The use of bone implements in the Old Paleolithic period ", Antiquity, vol. 22, p. 36-57, 1938.

36. Pierre Teilhard de Chardin, " Le "Sinanthropus » de Pékin, état actuel de nos connaissances sur le fossile et son gisement ", L'Anthropologie, t. XLI, no 1-2, 1931, p. 1-11.

37. Sabine Gaudzinski, Elaine Turner, Anna Paola AnzideI, Esteban Álvarez Fernández, JoAquin Arroyo Cabrales, Jacques CinQ-Mars, Viola T. Dobosi, Adrien Hannus, Eileen Johnson, Suzanne C. Münzel, Anne ScheER et Paula ViLla, "The use of proboscidean remains in everyday Palaeolithic life ", Quaternary International, $\mathrm{n}^{\circ}$ 12-128, 2005, p. 179-194; Giovanni BoschiaN et Daniela SACCÀ, «In the elephant, everything is good : Carcass use and re-useat Castel di Guido (Italy) ", Quaternary International, $\mathrm{n}^{\circ} 361,2015$, p. 288-296.

38. Mathieu LeROYER, Palethnologie acheuléenne: de la technologie bifaciale à l'organisation de la subsistance collective : étude du site de Boxgrove - EarthamPit (West Sussex, Angleterre) et de deux sites du cours moyen de la Seine, thèse de doctorat, université de Paris 1 Panthéon-Sorbonne, Paris, 2016.

39. Italo Biddittu et Aldo Giacomo Segre, "Utilizzazione dell'osson el Paleolitico inferiore italiano " in Atti XXIII riunione scientifica dell'Istituto Italiano di Preistoria e Protostoria nel Lazio (7-9 Maggio 1980), 1982, p. 89-104; Cesare Pitti et Antonio Mario Radmilli, « La stazione del Paleolitico inferiore a Castel di Guido presso Roma ", in Atti della XXIV riunione scientifica dell'Istituto Italiano di Preistoria e Protostorian el Lazio, 8-11 oct. Firenze 1982, 1984, p. 119-129.

40. Mathieu Leroyer, op. cit., 2016.

41. Italo Biddittu et Aldo Giacomo Segre, "Utilizzazione dell'osson el Paleolitico inferior italian », op. cit.
42. Ursula MANiA, «The utilisation of large mammal bones in Bilzingsleben - a special variant of Middle Pleistocene man's relationship to his environment ", in Herberth Ullrich (dir.), Man and Environment in the Palaeolithic, colloque international de Liège, Liège (2-7 mai 1993), Études et recherches archéologiques de l'université de Liège, 62, 1995, p. 239-246.

43. Marie-Anne Julien, Bruce HARDy, MareikeC. Stahlschmidt, Brigitte Urban, Jordi Serangeli et Nicholas J. CONARD, «Characterizing the Lower Paleolithic bone industry from Schöningen 12 II: A multi-proxy study ", Journal of Human Evolution, $\mathrm{n}^{\circ} 89,2015$, p. 264-286.

44. Jordi Rosell, Ruth Blasco, Gerard Campeny, Juan Carlos Díez, Rodrigo A. Alcalde, Leticia MenéndeZ, Juan Luis Arsuaga, José M. Bermúdez de Castro et Eudald Carbonell, «Bone as a technological raw material at the Gran Dolina site (Sierra de Atapuerca, Burgos, Spain) », Journal of Human Evolution, vol. 61, n 1, 2011, p. 125-131.

45. Viola T. DoвosI, « Ex Proboscideis- proboscidean remains as raw material at four Palaeolithic sites, Hungary ", in Giuseppe CAvarRetTA, Patrizia Gioia, Margherita Mussi et Maria Rita Рацомво (dir.), The world of elephants, Proceedings of the First International Congress, Rome, Consiglio Nazionale Delle Ricerche, 2001, p. 429443.

46. Hartmun Thieme, « Lower Paleolithic hunting spears from Germany », Nature, ${ }^{\circ} 385$, 1977, p. 807-810.

47. Werner H. Sсносн, Gerlinde BigGa, Utz BöHner, Pascale Richter, Thomas Terberger, "New insights on the wooden weapons from the Paleolithic site of Schöningen ", Journal of Human Evolution, $\mathrm{n}^{\circ}$ 89, 2015, p. 214-225.

48. Kenneth P. OAKLeY, Peter Andrews, Lawrence H. KeELEY, J.-Desmond ClARK, « A reappraisal of the Clacton spear point », Proceedings of Prehistory Society, $\mathrm{n}^{\circ} 43,1977$, p. 13-30.

49. Joachim HaHN, « Les ivoires en Allemagne : débitage, façonnage et utilisation au Paléolithique supérieur ", in Joachim HaHN, Michel MENU, Yvette TABorin, Philippe WALter et François Widemann (dir.), Le travail et l'usage de l'ivoire au Paléolithique supérieur, Actes de la Table Ronde, Ravello, 29-31 mai 1992, 1995, p. 115-136; Despina Liolios, Variabilité et caractéristiques du travail des matières osseuses au début de l'Aurignacien : approche technologique et économique, thèse de doctorat d'ethnologie et de sociologie comparative, université Paris 10, Nanterre, Paris, 1999.

50. Élise Tartar, De l'os à l'outil, op. cit.; Élise Tartar, Nicolas Teyssandier, François Bon et 
Despina Liolios, op . cit., 2006; José-Miguel Tejero, « Towards Complexity in Osseous Raw Material Exploitation by the First Anatomically Modern Humans in Europe: Aurignacian Deer Antler Working ", Journal of Anthropological Archaeology, $\mathrm{n}^{\circ}$ 36, 2014, p. 72-92; Marie-Cécile Soulier, Nejma Goutas, Christian Normand, Alexandra LEGRAND et Randall White, "Regards croisés de l'archéozoologue et du technologue sur l'exploitation des ressources animales à l'Aurignacien archaïque : l'exemple d'Isturitz (PyrénéesAtlantiques, France) ", in Céline Thí́BAut, Sandrine Costamagno et Émilie Claud (dir.), Exploitation des ressources organiques à la fin $d u$ Paléolithique moyen et au début du Paléolithique supérieur : interactions entre environnement et comportements techniques, $\mathrm{XXVII}{ }^{\mathrm{e}}$ Congrès Préhistorique de France, 31 mai-5 juin 2010, Mémoire de la Société Préhistorique Française, vol. 2, 2014, p. 315-332.

51. Despina Liolios, Variabilité et caractéristiques du travail des matières osseuses, op. cit., 1999; Francesco d'Errico, Michèle Julien, Despina Liolios, Marian VAnHaeren et Dominique BAFFIER, " Many awls in our argument. Bone tool manufacture and use in the Châtelperronian and Aurignacian levels of the Grotte du Renne at Arcy-sur-Cure ", in João ZILHÃo et Francesco d'Errico (dir.), The Chronology of the Aurignacian and of the Transitional Technocomplexes: Dating, Stratigraphies, Cultural Implications, Proceeding of Symposium 6.I of the XIV th Congress of the UISPP (University of Liège, Belgium, September 2-6, 2001), Trabalhos de Arqueologia 33, Lisbonne, 2003, p. 247-270; Camille Anne Jequier, Matteo RomAndini et Marco PEREsAnI, « Les retouchoirs en matières dures animales : une comparaison entre Moustérien final et Uluzzien ", Comptes Rendus Palevol, vol. 11, no 4, 2012, p. 283-292.

52. Voir Jean-Jacques Hublin, « Les restes humains ", in Marcel Otтe (dir.), Les Aurignaciens, op. cit., p. 95-112; Christine Verna, Véronique DUJARdin et Erik Trinkaus, " The Early Aurignacian human remains from La Quina-Aval (France) ", Journal of Human Evolution, vol. 62, n 5, 2012, p. 605-617.

53. Anne VINCENT, « L'os comme artefact au Paléolithique moyen : principe d'étude et premiers résultats », op. cit., 1988; Anne VINCENT, L'outillage osseux au Paléolithique moyen : une nouvelle approche, op. cit. 1993; Élise TARTAR et Sandrine Costamagno, " L'utilisation des matières osseuses au Moustérien », in Alain Turq, Jean-Philippe FaIvre, Bruno Maureille, Christelle Lahaye et Priscilla BAyle (dir.), Néandertal à La Loupe, catalogue d'exposition du musée National de Préhistoire, Les Eyzies, 2016, p. 89-96.
54. Élise TARTAR, « Réflexion autour de la fonction des retouchoirs en os de l'Aurignacien ancien ", Bulletin de la Société préhistorique française, vol. $109, \mathrm{n}^{\circ} 1,2012$, p. 69-83.

55. Pour les périodes plus récentes, à l'Aurignacien, ces outils pourraient aussi avoir servi de percuteurs pour le débitage de lamelles lithiques, voir ibid., p. 71.

56. Léon Henri-Martin, Recherches sur l'évolution du Moustérien dans le gisement de la Quina (Charente), t. 2 : industrie lithique, Angoulême, Mémoires de la Société Archéologique et Historique de la Charente, no 14, 1923; Catherine Schwab, "Éclats diaphysaires du Paléolithique moyen et supérieur : la grotte d'Isturitz (PyrénéesAtlantiques) », in Marylène Patou-Mathis (dir.), "Fiches typologiques de l'industrie osseuse préhistorique. Commission de nomenclature sur l'industrie de l'os préhistorique. Cahier X: Os à impressions et éraillures », Treignes, CEDARC, 2002, p. 59-74; JeanChristophe CAstel, François-Xavier Chauvière et Stéphane Madeleine, "Sur os et sur dents : Les "retouchoirs" aurignaciens de La Ferrassie (Savignac-de-Miremont, Dordogne) ", Paléo, vol. 15, 2003, p. 29-50; Élise TARTAR, « Réflexion autour de la fonction des retouchoirs en os de l'Aurignacien ancien », op. cit.

57. Matteo Romandini, Emanuela Cristiani et Marco Peresani, «A retouched bone shaft from the Late Mousterian at Fumane cave (Italy). Technological, experimental and microwear analysis ", Comptes Rendus Palevol, vol. 14, 2015, p. $63-72$.

58. Jordi Rosell et al., «Bone as a technological raw material at the Gran Dolina site (Sierra de Atapuerca, Burgos, Spain) ", Journal of Human Evolution, vol. 61, n 1, 2011, p. 125-131.

59. Millán Mozota Holgueras, « El utillaje óseo musteriense del nivel " $\mathrm{D}$ " de Axlor (Dima, Vizcaya): análisis de la cadena operativa », Trabajos de Prehistoria, vol. 66, n 1, 2009, p. 27-46.

60. Ariane Burke et Francesco d'Errico, "A Middle Palaeolithic bone tool from Crimea (Ukraine) », Antiquity, vol. 82, 2008, p. 843-852.

61. Sabine GAUdzINSKI, « Middle Palaeolithic Bone Tools from the Open-Air Site SalzgitterLebenstedt (Germany) ", Journal of Archaeological Science, vol. 26, 1999, p. 125-141.

62. Marie Soressi, Shannon P. McPherron, Michel LenoIr, Tamara Dogandzic, Paul Goldberg, Zenobia Jacobs, Yolaine MaIgrot, Naomi L. Martisius, Christopher E. Miller, William Rendu, Michael Richards, Matthew M. Skinner, Teresa E. Steele, Sahra Talamo et Jean-Pierre TexiER, "Neandertals made the first specialized bone tools of Europe », Proceedings of 
the National Academy of Sciences of the U.S.A, $\mathrm{n}^{\circ} 110$, 2013, p. 14186-14190.

63. Katerina Douka et Enza Elena SpInApolis, " Neanderthal Shell Tool Production: Evidence from Middle Palaeolithic Italy and Greece ", Journal of World Prehistory, $\mathrm{n}^{\circ}$ 25, 2012, p. 45-79; Francesca Romagnoli, Javier Baena, Ana Isabel Pardo Naranjo et Lucia Sarti, " Evaluating the performance of the cutting edge of Neanderthal shell tools: A new experimental approach. Use, mode of operation, and strength of Callistachione from a behavioural, Quina perspective ", Quaternay International, vol. 427, part. B, 2017, p. 216-228.

64. José-Miguel Tejero, « Towards Complexity in Osseous Raw Material »,op. cit., 2014.

65. Ibid., 2014.

66. Olaf Jöris et Martin StreEt, « At the end of the 14C time scale - The Middle to Upper Palaeolithic record of western Eurasia ", Journal of Human Evolution, vol. 55, 2008, p. 782-802; William E. BANKs, Francesco d'ERrico et João ZILHÃO, " Human climate interaction during the Early Upper Paleolithic: testing the hypothesis of an adaptive shift between the Proto-Aurignacian and the Early Aurignacian ", Journal of Human Evolution, vol. 64, 2013, p. 39-55.

67. François BoN, " A brief overview of Aurignacian cultures in the context of the industries of the transition from the Middle to Upper Paleolithic », in Ofer Bar-Yosef et João ZiLHão (dir.), Towards a definition of the Aurignacian, proceedings of the symposium held in Lisbon, Portugal, June 25-30, 2002, Trabalhos de arqueologia 45, Lisbonne, 2006, p. 133-144; Nicolas TEYSSANDIER, François Bon et Jean-Guillaume BORDES, « Within projectile range: some thoughts on the appearance of the Aurignacian in Europe ", Journal of Anthropological Research, vol. 66, 2010, p. 209-229; José-Miguel Tejero, op. cit.; JoséMiguel Tejero et Stefano Grimaldi, " Assessing bone and antler exploitation at Riparo Mochi (Balzi Rossi, Italy): implications for the characterization of the Aurignacian in south-western Europe ", Journal of Archaeological Science, vol. 61, 2015, p. 59-77.

68. José-Miguel Tejero, « Towards Complexity in Osseous Raw Material »,op. cit.

69. Les plus anciens vestiges d'homme moderne en Europe sont, pour le moment, connus à partir de l'Aurignacien ancien, voir Jean-Jacques Hublin, «Les restes humaines », op. cit.; Christine Verna, Véronique Dujardin et Erik Trinkaus, The Early Aurignacian human remains from $\mathrm{La}$ Quina-Aval (France), op. cit.

70. Francesco d'Errico, Michèle Julien, Despina Liolios, Marian VANHAeren et
Dominique BAfFIER, " Many awls in our argument. Bone tool manufacture and use in the Châtelperronian and Aurignacian levels of the Grotte du Renne at Arcy-sur-Cure », op. cit.; François Caron, Francesco d'Errico, Pierre Del Mora, Frédéric Santos et João Zilhão, "The Reality of Neandertal Symbolic Behavior at the Grotte du Renne, Arcy-sur-Cure, France », PLoS ONE, vol. 6, no 6, e21545. doi : 10.1371/journal. pone. 0021545

71. Randall WhITE, « Personal Ornaments from the Grotte du Renne at Arcy-sur-Cure ", Athena Review, vol. 2, n 4, 2001, p. 41-46; Thomas Higham, Roger Jacobi, Michèle Julien, Francine David, Laura BASELL, Rachel Wood, William Davies et Christopher BronKs RAMSEY, "Chronology of the Grotte du Renne (France) and implications for the context of ornaments and human remains within the Châtelperronian ", Proceedings of the National Academy of Sciences, vol. 107, no 47, 2010, p. 2023420239.

72. Ludovic SlimaK, John Inge Svendsen, Jan Mangerud, Hugues Plisson, Herbjørn Presthus Heggen, Alexis Brugère et Pavel Yurievich PAvlov, " Late Mousterian Persistence near the Arctic Circle ", Science, vol. 332, n 6031, 2011, p. $841-845$.

73. Nicolas Zwyns, Wil RoEbroeks, Shannon P. McPherron, Adam JAgich et Jean-Jacques Hublin, "Comment on "Late Mousterian Persistence near the Arctic Circle" ", Science, vol. 335, $\mathrm{n}^{\circ}$ 6065, 2012, p. 167; Ludovic SlimaK, John Inge Svendsen, Jan Mangerud, Hugues Plisson, Herbjørn Presthus Heggen, Alexis Brugère et Pavel Yurievich Pavlov, « Response to "Comment on Late Mousterian Persistence near the Arctic Circle" ", Science, vol. 335, n 6065, 2012, p. 167.

74. Claire Letourneux, Devine qui est venu diner à Brassempouy? Approche taphonomique pour une interprétation archéozoologique des vestiges osseux de l'Aurignacien ancien de la grotte des Hyènes (Brassempouy, Landes), thèse de doctorat, université de Paris 1, Panthéon-Sorbonne, 2003.

75. Aline Averboun, op. cit., 2000.

76. Élise TARTAR, De l'os à l'outil, op. cit.

77. Nejma Goutas, Caractérisation et évolution du Gravettien en France, op. cit., 2004.

78. Gaëlle Le Dosseur, La néolithisation au Levant sud à travers l'exploitation des matières osseuses. Étude techno-économique de onze séries d'industries osseuses du Natoufien au PPNB récent, thèse de doctorat, université de Paris 1 Panthéon-Sorbonne, 2006; Nejma Goutas, « Données inédites sur le Gravettien oriental : apport de la technologie osseuse à la caractérisation des occupations de Kostienki 4 (Alexandrovska, région de Voronej, Russie) », Bul- 
letin de la Société Préhistorique Française, vol. 112, $\mathrm{n}^{\circ} 4$, octobre-décembre 2015, p. 647-692.

79. Gerd AlbRecht, « Testing of materials as used for bone points of the Upper Palaeolithic », in Henriette Camps-Fabrer (dir.), Méthodologie appliquée à l'industrie de l'os préhistorique, deuxième colloque international sur l'industrie de l'os dans la Préhistoire, Abbaye de Sénanque (Vaucluse), 9-12 juin 1976, Partis, éditions du CNRS, 1977, p. 119-124; Roger D. GUthrie, "Osseous projectile points : biological considerations affecting raw material selection and design among paleolithic and paleoindian people", in Juliet CLUTTON-BRocK et Caroline Grigson (dir.), Animals and archaeology, I : hunters and theirprey, Oxford, Archaeopress, BAR International Series, 165, 1983, p. 273-294.

80. Sensu Marie-Louise InIZAN, Michèle Reduron, Jacques Tixier et Hélène Roche, Préhistoire de la pierre taillée 4, Paris, C.R.E.P, 1995.

81. Heidi KNECHT, « Projectile points of bone, antler, and stone. Experimental explorations of manufacture and use ", in Heidi KNECHT (dir.), Projectile Technology, New York, Plenum Press, 1997, p. 191-212; Despina Lıolıos, op. cit.; JoséMiguel Tejero, Marianne Christensen et Pierre BoDU, " Red deer antler technology and early modern humans in Southeast Europe ", op. cit.; Marie-Cécile Soulier, Nejma Goutas, Christian Normand, Alexandra Legrand et Randall WHITE, op. cit.

82. Élise Tartar, com. pers.

83. José-Miguel Tejero, Marianne Christensen et Pierre Bodu, op. cit.

84. Despina Liolios, op. cit.

85. Voir détails in Nejma GoutAs, « Réflexions sur une innovation technique gravettienne importante : le double rainurage ", Bulletin de la Société préhistorique française, vol. 106, n 3, 2009, p. 437456.

86. Nejma Goutas, op. cit., 2004.

87. Voir Michele LANGley (dir.), Osseous Projectile Weaponry: Towards an Understanding of Pleistocene Cultural Variability, Cambridge, Cambridge University press, 2016.

88. Joachim HAHN, "Schlaginstrumente des Aurignacien aus dem Geissenklösterle bei Blaubeuren, Alb-Donau-Kreis », Archäologie Korrbl, vol. 14, 1984, p. 351-355; Leif STEGUWEIT et Gerhard TRNKA, "Ivory artefacts from the Aurignacian site Alberndorf I in the Pulkauvalley (LowerAustria) and their interpretation as tools ", Wissenschaftliche Mitteilungen aus dem Niederösterreichischen, vol. 19, 2008, p. 149-165.

89. Nejma Goutas, " From stone flaking to grinding: Three original Pavlovian antler tools from Moravia (Pavlov I, Czech Republic) ",
Quaternary International, vol. 359-360, 2015, p. 240260.

90. François Bordes, «Percuteur en bois de renne du Solutréen supérieur de Laugerie-Haute Ouest », in Henriette Camps-Fabrer (dir.), Premier colloque international sur l'industrie de l'os dans la Préhistoire, Abbaye de Sénanque, avril 1974, Aix-en-Provence, université de Provence, 1974, p. $97-100$.

91. Aline Averboun, «Un fragment de percuteur sur partie basilaire de la grotte magdalénienne d'Enlène (Ariège) ", Bulletin de la Société préhistorique française, vol. 96, no 4, 1999, p. 497-504; Aline Averboun et Pierre Bodu, Fiche "Percuteur sur partie basilaire de bois de cervidé », in Marylène Patou-Mathis (dir.), Fiches typologiques de l'industrie osseuse préhistorique. Commission de nomenclature sur l'industrie de l'os préhistorique. Cahier $X$ : Os à impressions et éraillures, Treignes, CEDARC, 2002, p. 117-131; Malvina BaUmanN, À l'ombre des feuilles de Laurier, les équipements osseux solutréens $d u$ sud-ouest de la France, thèse de doctorat, université de Paris 1 Panthéon-Sorbonne, Paris, 2014.

92. Jacques Pelegrin, "Sur une recherche technique expérimentale des techniques de débitage laminaire ", in Actes du colloque international. Expérimentation en archéologie. Bilan et perspectives. Archéologie expérimentale $2:$ La Terre, l'os et la pierre. La maison et les champs, Archéodrome de Beaune, avril 1988, Archéologie aujourd'hui, Paris, Errance, 1991, p. 118-128; Aline Averbouh et Pierre Bodu, op. cit., 2002.

93. Il s'agit d'une matière première utilisée pour tailler certaines feuilles de lauriers.

94. Aline Averboun et Pierre Bodu, op. cit., 2002.

95. Malvina BaumanN, À l'ombre des feuilles de Laurier, op. cit.

96. Op. cit.

97. Assia Agoudjil, Essai de caractérisation des industries en matières dures animales solutréennes. Apport de l'étude du niveau solutréen moyen (couche $H$ » à feuilles de laurier) de Laugerie-Haute Ouest à la connaissance des modalités de débitage $d u$ bois de cervidé, mémoire de DEA, université de Paris 1 Panthéon-Sorbonne, 2005; Malvina BaumanN, op. cit.

98. Pierre Cattelain, « Un crochet de propulseur solutréen de la grotte de Combe Saunière I (Dordogne) ", Bulletin de la Société préhistorique française, vol. 86, no 7, 1989, p. 213-216; Pierre Cattelain, "Propulseurs magdaléniens : marqueurs culturels régionaux ", in Véronique DUJARDIN (dir.), Industrie osseuse et parures $d u$ Solutréen au Magdalénien en Europe. Actes de la table ronde sur le Paléolithique supérieur récent, 
Angoulême (Charente), 28-30 mars 2003, Paris, Société Préhistorique Française, Mémoire 39, 2005, p. 301-317.

99. Danièle StORDEUR, Les aiguilles à chas au Paléolithique, Paris, CNRS, XIII ${ }^{e}$ supplément Gallia Préhistoire, 1979.

100. Le propulseur subsistera au Grønland, sur une grande partie du territoire américain, en Alaska, Micronésie et Australie, alors que l'aiguille à chas est utilisée dans la plupart des sociétés actuelles, partout dans le monde.

101. Pierre Cattelain, " Un propulseur inédit de la Grotte du Placard (Vilhonneur, Charente, France) provenant de la collection Breuil conservée à l'Institut de Paléontologie Humaine à Paris », Archéo-Situla, vol. 34, 2014, p. 41-42.

102. Pierre Cattelain, op. cit., 1989; Pierre Cattelain, "Un propulseur inédit de la grotte du Placard (Vilhonneur, Charente, France) », Notae Praehistoricae, $\mathrm{n}^{\circ}$ 24, 2004, p. 61-67; Pierre Cattelain, "Les propulseurs de l'Abri de La Madeleine (Dordogne, France) » in Jean-Jacques Cleyet-Merle, Jean-Michel Geneste, Elena MAN-Estier (dir.), L'art au quotidien - Objets ornés du Paléolithique supérieur (partie 3 - Techniques, supports et thèmes), Actes du colloque international, Les Eyzies-de-Tayac, 16-20 juin 2014, Paléo, numéro spécial, 2016, p. 217-242.

103. Mariana GvozDover, Art of the mammoth hunters. The finds from Avdeevo, Oxbow Monograph 49, P. Bahn, 1995.

104. Jacques Allain, Robert Fritsch, André Rigaud et Françoise Trotignon, " Le débitage $\mathrm{du}$ bois de renne dans les niveaux à raclettes du Badegoulien de l'Abri Fritsch et sa signification ", in HenrietteCAmps-Fabrer (dir.), Premier colloque international sur l'industrie de l'os dans la préhistoire, Abbaye de Sénanque (Vaucluse, 1974), Paris, éditions de l'université de Provence, 1975, p. 67-71; Aline Averboun et Jean-Marc Pétillon, "Identification of "debitage by fracturation" on reindeer antler : case study of the Badegoulian levels at the Cuzoul de Vers (Lot, France) ", in Justyna BAron et Bernadeta KufEL-DiaKowsKA (dir.), Written in Bones, Studies on Technological and Social Contexts of Past Faunal Skeletal Remains, Wroclaw, University of Wroclaw, 2011, p. 41-52; Jean-Marc Pétillon et Sylvain Ducasse, "From flakes to grooves: a technical shift in antler working during the Last Glacial Maximum in southwest France", Journal of Human Evolution, vol. $62, \mathrm{n}^{\circ} 4,2012$, p. 435-465.

105. Aline Averbouh, L'industrie en matières osseuses du Paléolithique, Document pour une caractérisation chrono-culturelle des principes de transformation et des productions associées, établi en vue de la sélection du matériel d'exposition des nouvelles salles du musée national de Préhistoire des Eyzies de Tayac, tapuscrit, 2002.

106. Danielle Stordeur, Les aiguilles à chas au Paléolithique, op. cit.

107. Aline Averboun, L'industrie en matières osseuses du Paléolithique, op. cit.; Pierre Cattelain, " Les propulseurs du Magdalénien moyen ancien et apparentés ", in Camille Bourdier, Lucie Chehmana, Romain Malgarini et Marta PoŁтоwicz-Вовак (dir.), L'essor du Magdalénien. Aspects culturels, symboliques et techniques des faciès à navettes et à Lussac-Angles, Actes de la séance de la Société préhistorique française (Besançon, 17-19 octobre 2013), Paris, Société préhistorique française, 2017, p. 235-248.

108. Aline Averboun, Technologie de la matière osseuse travaillée, op. cit.; id., "La production des équipements en matières osseuses à la Madeleine et à Laugerie-Basse ", Catalogue de l'exposition Grands sites d'Art magdalénien. La Madeleine et Laugerie-Basse il y a 15000 ans, MNP et MAAP, édition Réunion des Musées nationaux-Grand Palais, Les Eyzies-de-Tayac, 2014, p. 45-47.

109. Aline AverbouH, «La production des équipements en matières osseuses... », op. cit., 2014; id., "Utilisation et transformation des matières osseuses au Buisson-Campin (Verberie, Oise)/ Use and transformation of osseous material sat the Buisson Campin site (Verberie, Oise) », in Ezra Zubrow, Françoise Audouze et Jim Enloe (dir.), The Magdalenian Household, Unraveling Domesticity, Albany, Sunny Press, 2010, p. 76-90.

110. Valérie Feruglio, Fiche «Baguettes demirondes ", in Henriette CAMPS-FABRER (dir.), Fiches typologiques de l'industrie osseuse préhistorique, Commission de nomenclature sur l'industrie de l'os préhistorique, Cahier V: Bâtons percés, baguettes, Treignes, CEDARC, 1992, p. 71-83; Valérie Feruglio et Aline AverbouH, «Identification d'un marqueur identitaire des groupes du Magdalénien par l'approche technique, thématique et chronologique : l'exemple des baguettes demi-rondes à tubérosités ", in Jean-Jacques Cleyet-Merle, Jean-Michel Geneste et Elena MAn-Estier (dir.), L'art au quotidien. Objets ornés du Paléolithique supérieur, Actes du colloque international MNP Les Eyzies de Tayac, juin 2014, Paléo, sous presse.

111. Caractéristiques du Magdalénien moyen ancien.

112. Michèle JuLIEN, Les harpons magdaléniens, Paris, CNRS, Gallia Préhistoire, supplément 17, 1982.

113. Dominique Buisson et Aurélie Peltier, Fiche "Cuiller à cuilleron élargi » in Henriette CAMPs-Fabrer (dir.), Fiches typologiques de l'in- 
dustrie osseuse préhistorique, cahier VI, éléments récepteurs, Commission de nomenclature sur l'industrie de l'os préhistorique, Treignes, CEDARC, 1993, p. 141-143.

114. Aline Averboun, Technologie de la matière osseuse travaillée, op. cit.

115. Aline Averboun et Pierre Cattelain, « De l'analyse fonctionnelle des propulseurs paléolithiques par l'économie de leur production ", in Marylène Patou-Mathis, Pierre Cattelain et Denis Ramseyer (coord.), L'industrie osseuse pré- et protohistorique en Europe. Approches technologiques et fonctionnelles, Actes du XIV ${ }^{e}$ Congrès UISPP (2-8, septembre 2001, Liège), 2002, bulletin du Cercle archéologique Hesbaye-Condroz, vol. XXVI, p. 63-73.

116. Aline Averboun, Technologie de la matière osseuse travaillée, op. cit.

117. Aline Averboun, L'industrie en matières osseuses du Paléolithique, op cit.; id., " La production des équipements en matières osseuses du Paléolithique final au début du Mésolithique : approche synthétique de l'évolution des schémas de transformation ", in Aline Averboun, Peggy Jacquement-Bonnet et Jean-Jacques CleyetMerLe (dir.), L'Aquitaine à la fin des temps glaciaires, les sociétés de la transition du Paléolithique final au début du Mésolithique dans l'espace nord aquitain, hommage à Guy Célérier, juin 2014, musée national de Préhistoire, numéro hors-série Paléo, sous presse.

118. Jacques Pelegrin, « Les techniques de débitage laminaire au Tardiglaciaire : critères de diagnose et quelques réflexions ", in Boris VALENTIN, Pierre Bodu et Marianne Christensen (dir.), L'Europe centrale et septentrionale au Tardiglaciaire, Table ronde de Nemours (13-16 Mai 1997), Mémoires du musée de Préhistoire d'Ile de France 7, édition APRAIF, Nemours, 2000, p. 73-86.

119. Mathieu Seddas, Bilan sur les industries osseuses aziliennes d'Espagne et de France. La place des productions en matières dures animales $d u$ site de la Tourasse (Haute-Garonne) dans le contexte azilien des Pyrénées, mémoire de Master 1, université de Paris 1, Panthéon Sorbonne, 2012.

120. Mathieu SedDAs, L'exploitation $d u$ bois de cerf à l'Azilien de la grotte de Pégourié (Caniac-duCausse, Lot). Entre déchets et objets finis : témoins de déplacements programmés, mémoire de Master 2, université de Paris 1, Panthéon Sorbonne, 2013.

121. Marianne Christensen et André ChOllet, « L'industrie sur bois de cervidé et os des niveaux magdaléniens et aziliens du Bois-Ragot : étude préliminaire ", in André CHOLlet et Véronique Dujardin (dir.), La Grotte du Bois-Ragot (Vienne). Magdalénien et Azilien. Essai sur les hommes et leur environnement, Paris, Société préhistorique française, Mémoire de la Société Préhistorique Française, $n^{\circ}$ 37, 2015, p. 223-257.

122. Boris Valentin, op. cit.; François Bon, Préhistoire. La fabrique de l'homme, Paris, Seuil, 2009; Pierre NoIRET, « De quoi Gravettien est-il le nom? », in Marcel Otте (dir.), Les Gravettiens, op. cit., p. 28-64.

123. Boris Valentin, op. cit.; François Bon, op. cit.

124. Yvette TABorin, La Parure en coquillage au Paléolithique, Paris, CNRS, Gallia Préhistoire, supplément 29, 1993.

125. Marianne Christensen, L'industrie osseuse des chasseurs-cueilleurs, op. cit. ; Jean-Marc PÉtillon, «Vivre au bord du golfe de Gascogne au Paléolithique supérieur récent : vers un nouveau paradigme », in Catherine Dupont et Grégor MARChAND, Archéologie des chasseurs-cueilleurs maritimes. De la fonction des habitats à l'organisation de l'espace littoral, Actes de la séance de la Société préhistorique française (10-11 avril, Rennes), 2014, Paris, Société préhistorique française, vol. 6, 2016, p. 23-34. Publication en ligne : [www.prehistoire. org]. 